\title{
Robust stabilization for discrete-time Takagi-Sugeno fuzzy system based on N4SID models
}

Mohamed Ali Jemmali ${ }^{1}$, Martin J.-D. Otis ${ }^{2 *}$ and Mahmoud Ellouze ${ }^{3}$

1 University of Ottawa, Ontario, Canada, mjemm073@uottawa.ca

2* LAR.i Lab, University of Quebec at Chicoutimi, Quebec, Canada Martin Otis@uqac.ca

3 École Nationale d'Ingénieurs de Tunis, Université de Tunis El Manar, ellouze_mahmoude@yahoo.fr

\section{Abstract \\ Purpose}

Nonlinear systems identification from experimental data without any prior knowledge of the system parameters is a challenge in control and process diagnostic. It determines mathematical model parameters that are able to reproduce the dynamic behavior of a system. This paper combines two fundamental research areas: MIMO state space system identification and nonlinear control system. This combination produces a technique that leads to robust stabilization of a nonlinear Takagi-Sugeno fuzzy system (T-S).

Design/methodology/approach

The first part of this paper describes the identification based on the Numerical algorithm for Subspace State Space System IDentification (N4SID). The second part, from the identified models of first part, explains how we use the interpolation of Linear Time Invariants (LTI) models to build a nonlinear multiple model system, T-S model. For demonstration purposes, conditions on stability and stabilization of discrete time, Takagi-Sugeno (T-S) model were discussed.

Findings

Stability analysis based on the quadratic Lyapunov function to simplify implementation was explained in this paper. The LMIs (Linear Matrix Inequalities) technique obtained from the linearization of the BMIs (Bilinear Matrix Inequalities) was computed. The suggested N4SID2 algorithm had the smallest error value compared to other algorithms for all estimated system matrices.

Originality

The stabilization of the closed-loop discrete time T-S system, using the improved PDC control law (Parallel Distributed Compensation), was discussed to reconstruct the state from nonlinear Luenberger observers.

Keywords: Multiple model; Kalman filter; Takagi-Sugeno stabilization; PDC control law. 


\section{Introduction}

Recent research was developed in the context of the identification of multivariable systems. These researches, however, demonstrates robustness and effectiveness, not only on the theoretical aspect but also in real processes. Thus, complex systems are generally found in the industry, such as in the aluminum foundry, sawmill plant, robot manipulator (Costa et al., 2016), etc. The application of identification subspace algorithms in this area is promising for inverse diagnostic (Borjas and Garcia, 2011). In (Ma et al., 2018), large-scale wind power interfacing to the power grid was studied, and it presents the impact on the stability of the power system. However, with an additional damping controller of the wind generator, new ways for improving system damping and suppressing the low frequency oscillation (LFO) of power systems can be put forward. The N4SID was proposed to identify the state space model of the controlled object. In (De Simone and Guida, 2018), the N4SID method was used to obtain a dynamical model of the unmanned vehicle, while open-loop and closed-loop control algorithms were implemented on the ArduinoMega controller. In (Kishore et al., 2018), a closed-loop identification method for Multi-Input Multi-Output (MIMO) system has been proposed. The transfer function matrix of the dynamic system is identified in the form of state-space model using N4SID algorithm from the system. The identification in state space, especially for the N4SID algorithm, is widely used in many areas and for various applications. In particular, N4SID is exploited for estimating parameters of industrial systems, as for the detection and diagnostic analysis of electrical machines. A few studies are mentioned as shown below. The four studies presented in (Cao et al., 2014, Chadli et al., 2002, Chang and Yang, 2010, Euntai and Heejin, 2000) suggested using one of the most recognized algorithms which are: 1) Multivariable Output Error State Space (MOESP) firstly presented in (Hachicha et al., 2014), and 2) Canonical Variate Analysis (CVA) firstly presented in (Ichalal et al., 2014). The MOESP uses the techniques of linear algebra and geometry such as singular value decomposition (SVD), LQ factorization (L: Lower triangular matrix; Q: Orthogonal matrix), and matrices projection. This method involves determining the system matrices $\mathrm{A}$ and $\mathrm{C}$ directly from the extended observability matrix and determining the rest of the system matrices $\mathrm{B}, \mathrm{D}, \mathrm{Q}, \mathrm{R}$, and $\mathrm{S}$. This is done by using the least squares method (a similar approach is presented in (Krokavec and Filasova, 2014, Larimore, 1990, Hachicha et al., 2014). The CVA, however, is based on statistical arguments and it extensively uses angles and main directions.

In this paper, the identification method used is the Numerical algorithm for Subspace State Space System IDentification (N4SID) such as presented in (Ljung, 1999). N4SID is based on well-known linear algebra techniques, singular value decomposition, and QR decomposition (Q: orthogonal matrix; R: upper triangular matrix) for numerical implementation. It could estimate state space matrices of deterministic-stochastic systems directly from input and output data. N4SID is convergent and noniterative, which is numerically stable. Our work is based on a global modelling technique known as 
generic multiple model approach. The principle of this approach is aimed at reducing the complexity of the system by a decomposition of its operating space into a finite number of operating ranges. Indeed, a local lower-order linear model structure can often be used to describe the dynamic behavior of the system in this local area.

Therefore, one can suggest using multiple models to solve the whole system behavior. This method is well suited for modelling systems from experimental data. In this modelling approach, two families are known according to the structure used to interpolate the local models. The first, called TakagiSugeno multiple model, consists of local models sharing a single state vector. The second approach, decoupled multiple models, has each model with its own state vector. It has been sufficiently demonstrated by (Muhammad et al., 2013a, Muhammad et al., 2013b, van Overschee and de Moor, 1996, Prívara et al., 2010, Shafieezadeh-Abadeh and Kalhor, 2016, Söderström and Stoica, 1989), that the interest in the multiple model of Takagi-Sugeno is the synthesis of control law or estimating state of nonlinear systems. However, from a structural point of view, all these local models, constituting the multiple models, have the same sizes. Then, a single state vector is used. The complexity of these submodels is therefore constant regardless of the complexity of the system in different areas of operation. Most research has focused on T-S control such as Autonomous Vehicles control. Perform lane keeping under multiple constraints, roads with unknown curvature, and uncertain lateral wind force for autonomous vehicles is one of the trend and the challenge of research in our time (Nguyen et al., 2017b). We cite also (Nguyen et al., 2017a) who addresses the shared lateral control between a human driver and a lane keeping assist system of intelligent vehicles for both lane keeping and obstacle avoidance. In (Nguyen et al., 2018) and (Sentouh et al., 2018), the authors got a lot of attention in minimizing the conflict situations between these two driving actors. These authors avoid using quadratic Lyapunov function because of the conservatism seen in their results. Indeed, they used other function like nonquadratic Lyapunov function which is difficult to implement in real life and add more complexity to the studied system. The method of selection of Lyapunov function and the method of LMI's opening is a wonderful field of research which has led to great interest by researchers. In (Zhang et al., 2018b) the authors investigated the sliding-mode control problem of T-S fuzzy multiagent system. Therefore, a cooperative fuzzy-based dynamical sliding-mode controller was designed. The main idea was to transform the fuzzy weighting matrix into a set of scalars by solving LMI's. Furthermore, the energy-coast was studied by using the linear quadratic regulator. We mention as well, the problem of quantized feedback control of nonlinear Markov Jump Systems (MJS's) demonstrated in (Zhang et al., 2018a). Based on T-S fuzzy model, the nonlinear plant was represented by a class of fuzzy (MJS's) with timevarying delay. The quantized states was utilized for the control aim. The sector bound approach was exploited to deal with quantization errors. The Lyapunov function depended both on information and fuzzy basis functions. On the other hand, in our paper, we present an improved PDC control law for the T-S system to minimize the conservatism and give more relaxation for the results. 
The efficiency and the modelling accuracy of this new multiple model presentation, recorded in simulation, incited us to evaluate its potential on simulation example.

The contribution of this paper is related to the design of a new algorithm which combines these two methods previously described. Individually, both methods have very successful results for the robust control of nonlinear systems described by multiple models from N4SID algorithm. Then, our paper suggests improving their performance by this combination. We also compared the N4SID and the MOESP algorithms in order to know how our improvement increases the algorithm performances.

The paper is organized as follows. Firstly, a review was done on the main algorithms in this field's sub-space N4SID method with other approaches such as polynomial identification, iterative, and even with other sub-space method. Then, the second part presents the selected algorithm for our application, N4SID model, and demonstrates two variations, N4SID 1 and 2, which will be evaluated and tested. The third section of the article is devoted to the presentation of the multi-model control. The following section focuses on the study of the stabilization of Takagi-Sugeno fuzzy system (Prívara et al., 2010, Sofianos and Boutalis, 2014, Tanaka et al., 1998), based on improved Parallel Distributed Compensation (PDC) control law. Finally, the last part of this paper is dedicated to the synthesis of nonlinear observer based on interpolation of linear observer of Luenberger type called multiobserver.

\section{Related work}

Systems identification literature is mainly concerned with computing polynomial models. This method is effective in the case of Single Input Single Output (SISO) systems represented by a linear transfer function. In the case of Multi-input Multi-output (MIMO) systems, when calculating the polynomial model, we are faced with unsolvable mathematical situations. These situations are, however, known to typically give rise to numerically ill-conditioned mathematical problems. Therefore, recent research works have proposed different approaches to solve this issue and minimize the parameterization required. These approaches are called Subspace methods which are described in the following sections.

\section{Iterative identification model}

The classical identification algorithms proposed by (Ljung, 1999, Söderström and Stoica, 1989, Yu et al., 2018) were a solution for the identification of multivariable systems. However, this method diverges sometimes, as it is an iterative algorithm. Also, iterative algorithms require knowledge of the order, and the observability or controllability indicates that classical algorithms need a parameterization and nonlinear optimizations. To avoid divergence problems, the research works are focused on the non-iterative methods such as N4SID from (Ljung, 1999), and also MOESP algorithms presented in (Hachicha et al., 2014). They are both based on geometry and linear algebra and are explained in the next section. 


\section{Subspace identification model}

This model was presented to be a powerful alternative to the classical system identification method based on iterative approaches. The key step of this method is the oblique projection of subspaces generated by the block Hankel matrices formed by input and output data of the system. Indeed, in practice, methods of subspaces, whose name reflects that linear models can be obtained from subspaces of certain matrices, is computed from data inputs and outputs. Then, only the order of the system is needed and it can be determined through input-output, meaning through inspection of the dominant singular value. Moreover, N4SID doesn't need an extra parameterization of the initial state when estimating a state space from data measured on a plant with a non-zero initial condition.

N4SID and MOESP algorithms are similar. However, the difference is in the numerical implementation level. N4SID use the QR decomposition and MOESP use the LQ decomposition. There is also a difference between these algorithms in the extraction of the system matrices and the convergence speed in addition to the level of the estimation error. Another difference between these two algorithms is related to the kind of employed subspaces projections in the Hankel matrices. These matrices are obviously constituted of the system input/output data. In other words, N4SID uses oblique projection while MOESP is particularly based on the orthogonal projection.

Many researches confirm this difference between these two algorithms. For proof purpose we compute the performance indicators usually used to validate a model. They are the mean relative square error (MRSE) and mean related variance (MVAF). These two indicators determine the estimation error and evaluate the accuracy identification between algorithms. This method determines a criterion to measure the distance between the model and the real system. To sum up, we can determine the right choice based on the system aspect and the desired performance like processing time and cross validation (Borjas and Garcia, 2011).

\section{Contribution}

N4SID algorithm and multiple model approach integration, in one algorithm to control a nonlinear multivariable system, should be reflected in an increase of the robustness. The algorithm identifies multi inputs multi outputs (MIMO) systems that are modelled by a state space representation. The algorithm reliably considers the parameters of a deterministic-stochastic, dynamic and linear time invariant multivariable system. It is known that this estimate gives accurate parameters of the process. N4SID is a convergent algorithm and is simple to implement. Our algorithm will be used with a nonlinear system in a local area, around an operating point. Multiple model approach will interpolate this local area to cover all nonlinear area. The combined result would yield an improved nonlinear control. As suggested in the next section, this paper is the first that combines these two robust methods to get an efficacy and new technique of nonlinear stabilization. Firstly, we present a review of the N4SID 
method. Secondly, we explain the theoretical control part and finally, we validate our model by numerical simulations.

\section{Model description}

Given $\mathrm{N}$ measurements of the inputs and the outputs generated by the combined stochastic and deterministic system of order n, we considered the following model for identification:

$$
\begin{gathered}
x(k+1)=A x(k)+B u(k)+w(k), \\
\mathrm{y}(\mathrm{k})=\mathrm{Cx}(\mathrm{k})+\mathrm{Du}(\mathrm{k})+\mathrm{v}(\mathrm{k}),
\end{gathered}
$$

where $A \in R^{n x n}$ is the state matrix, $B \in R^{n x m}$ is the control matrix, $C \in R^{l x n}$ is the observation matrix, $D \in R^{l x m}$ is the direct transmission matrix. $v \in R^{l x l}$ and $w \in R^{n x l}$ are both zero-mean Gaussian white noise.

\subsection{Objective of N4SID method}

From the control vectors $u=\left[u_{1} u_{2} \cdots u_{n}\right]$; and measurement vectors $y=\left[y_{1} y_{2} \cdots y_{n}\right]$ we want to determine the order $\mathrm{n}$ of the unknown system, the system matrices $A, B, C, D$ and the noise matrix $Q^{s}, S^{s}, R^{s}$ E denotes the expected value as operator and $\delta_{k l}$ the Kornecker delta. Then we can calculate:

$$
\mathrm{E}\left[\left(\frac{w_{\mathrm{k}}}{v_{\mathrm{k}}}\right)\left(\mathrm{w}_{\mathrm{l}}^{\mathrm{t}} \mathrm{v}_{\mathrm{l}}^{\mathrm{t}}\right)\right]=\left[\begin{array}{ll}
\left(\mathrm{Q}^{\mathrm{s}}\right) & \left(\mathrm{S}^{\mathrm{s}}\right) \\
\left(\mathrm{S}^{\mathrm{s}}\right)^{\mathrm{T}} & \left(\mathrm{R}^{\mathrm{s}}\right)
\end{array}\right] \delta_{\mathrm{kl}} \geq 0^{2}
$$

\subsection{Decomposition of deterministic-stochastic models}

State space representation can be decomposed into two sub-models, deterministic sub-model and stochastic sub-model. Let $x_{k}^{d}, x_{k}^{s}$ are respectively the deterministic and stochastic components of the state vector represented in equation (4) and (5). Also, $y_{k}^{d}, y_{k}^{s}$ are respectively the deterministic and stochastic components of the measurement vector presented in (5).

$$
\begin{aligned}
& x_{k}=x_{k}^{d}+x_{k}^{s} \\
& y_{k}=y_{k}^{d}+y_{k}^{s}
\end{aligned}
$$

However, the system can be decoupled into deterministic and stochastic parts defined as follow:

$$
\begin{gathered}
\left\{\begin{array}{c}
x_{k+1}^{d}=A x_{k}^{d}+B u_{k} \\
y_{k}^{d}=C x_{k}^{d}+D u_{k}
\end{array},\right. \\
\left\{\begin{array}{c}
x_{k+1}^{s}=A x_{k}^{s}+w_{k} \\
y_{k}^{s}=C x_{k}^{s}+v_{k}
\end{array}\right.
\end{gathered}
$$


It is assumed that the pair $(A, C)$ is observable, and the pair $A, B\left(Q^{s}\right)^{\frac{1}{2}}$ is controllable. The controllable pair $(A, B)$ can be stable or unstable. However, the controllable modes $A,\left(Q^{s}\right)^{\frac{1}{2}}$ must be stable. We also assume that the stochastic state $x_{k}^{S}$ and stochastic output $y_{k}^{s}$ are quasi-stationary. Finally, using this assumption, we can then, in the next section, represent the N4SID algorithm.

\subsection{N4SID algorithm}

In this section, we present two N4SID approach (N4SID 1 and 2 in the following) which the main difference is: N4SID 2 has the same steps as N4SID 1 except that it uses the approximate state $\widetilde{X}_{l}$ and all the systems matrices. This algorithm determines the state directly from the input and output data and minimizes the number of equations.

First, the N4SID 1 algorithm is explained in Figure 1. We have therefore, from a finite number of input-output data $\left(U_{k}, Y_{k}\right)$, the possibility to form the Hankel matrices (defined in appendix A) $\left(Y_{p}, Y_{f}\right)^{T}$ and $\left(U_{p}, U_{f}\right)^{T}$. In addition, we deduce the oblique projections $\left(Z_{i}, Z_{i-1}\right)$.
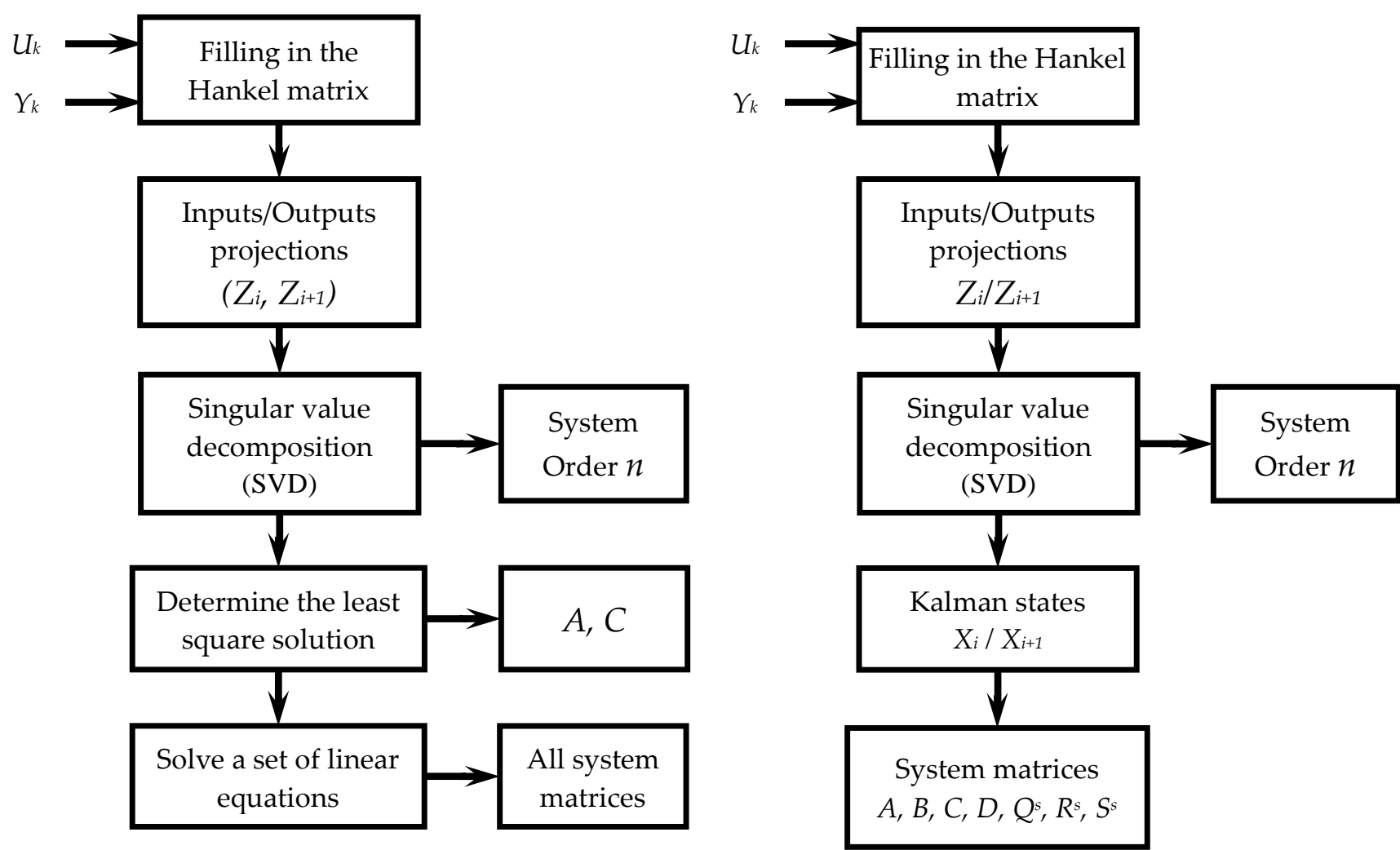

Figure 1. Steps of the N4SID1 algorithm.

Figure 2. Steps of the N4SID2 algorithm. 
These projections are useful in determining the combined system. Indeed, the linear combinations to be made from the input-output block Hankel matrices to generate $\left(Z_{i}, Z_{i-1}\right)$ (projections defined in the appendix C) are functions of the system matrices. Moreover, the system matrices can be retrieved from these linear combinations, as shown in the appendix C.

From input-output we can define the extended observability matrix $\Gamma_{i}$. Trough $\Gamma_{i}=$ $\left[\begin{array}{llll}C & C A & \ldots & C A^{i-1}\end{array}\right]$, we are able find the $A$ and $C$ matrices:

- Matrix C: If we show the extended observability matrix, it is important to note that we can extract directly from the first row of $\Gamma$ the $\mathrm{C}$ matrix.

- Matrix A: It is determined from the shift structure of $\Gamma_{i}$, denoting $: \overline{\Gamma_{i}}=\underline{\Gamma_{i}} A$; where $\underline{\Gamma_{i}}$ is the matrix $\Gamma_{i}$ without the last 1 rows: $\underline{\Gamma_{i}}=\left(\begin{array}{lllll}C & C A & \ldots & C A^{i-2}\end{array}\right) . \overline{\Gamma_{i}}$ is without the first 1 rows: $\overline{\Gamma_{i}}=$ $\left(\begin{array}{llll}C A & C A^{2} & \ldots & C A^{i-1}\end{array}\right)$. Therefore, the matrix $\mathrm{A}$ is expressed as: $A=\overline{\Gamma_{i}^{+}} \underline{\Gamma_{i}}$. Later then, we determine the rest of system matrices $B, D, Q^{s}, R^{s}$ and $S^{s}$ from linear regression algorithm and the resolution of linear combination demonstrated in appendix $\mathrm{C}$.

This means N4SID 2 does not need to go through the complicated step 4 shown in Figure 1. However, it is clearly explained in the appendix $C$ for determining B and D matrices as shown in Figure 2. Also, the Kalman states are determined as it is explained in (Verhaegen, 1994).

\section{Multiple model control}

A multiple model or T-S (Takagi-Sugeno) model is a nonlinear system in the form of linear interpolation of $\mathrm{n}$ premises. The three methods used for obtaining the multiple model representation are: 1 ) the identification technique, 2) linearization around different operating points, and 3) polytopic transformation. In the first step, we identify the model parameters based on the given input-output. The second and third steps are assumed to have a nonlinear mathematical model.

Conditions on stability and stabilization of discrete-Time T-S (Takagi-Sugeno) systems will be shown in the next section. Stability analysis is derived via quadratic Lyapunov function method and LMIs (Linear Matrix inequalities) formulation, obtained from BMIs (Bilinear Matrix Inequality). Discrete-Time T-S systems using improved PDC (Parallel Distributed Compensation) technique was applied in order to estimate state with the multi-observer technique..

\subsection{Discret Takagi-Sugeno system}


We considered a Takagi-Sugeno (T-S) model described by fuzzy IF-THEN rules. The ith rule of the model is of the following form:

\section{Rule i}

IF $F_{1}(k)$ is $M_{1 i}$ and $\ldots F_{n}(k)$ is $M_{n i}$ THEN

$\left\{\begin{array}{c}x(k+1)=A_{i} x(k)+B_{i} u(k) \\ y(k)=C x(k)\end{array}\right.$,

where $M_{1 \mathrm{i}}, \ldots . M_{n i}$ are the fuzzy sets and $n$ is the number of the rules. In addition, $x_{k} \in \mathbb{R}^{n}$ is the state vector; $u_{k} \in \mathbb{R}^{p}$ is the measurable output vector; $A_{i}, B_{i}$ and $C$ are the system matrices with appropriate dimension. Then, the premise variables are $Z(k)=\left[Z_{1}(k) \ldots . \quad Z_{q}(k)\right]$.Therefore, a T-S model is based on the interpolation between several LTI local models using the following equations:

$$
\begin{aligned}
\mathrm{x}(\mathrm{k}+1)= & \sum_{\mathrm{i}=1}^{\mathrm{n}} \mu_{\mathrm{i}}(\mathrm{Z}(\mathrm{k}))\left(\mathrm{A}_{\mathrm{i}} \mathrm{x}(\mathrm{k})+\mathrm{B}_{\mathrm{i}} \mathrm{u}(\mathrm{k})\right) \text { and } \\
& \left\{\begin{array}{c}
\sum_{\mathrm{i}=1}^{\mathrm{n}} \mu_{\mathrm{i}}(\mathrm{Z}(\mathrm{k}))=1 \\
\mu_{\mathrm{i}}(\mathrm{Z}(\mathrm{k})) \geq 0
\end{array}\right. \text { with, } \\
\mu_{\mathrm{i}}(\mathrm{Z})= & \frac{\mathrm{w}_{\mathrm{i}}(\mathrm{Z}(\mathrm{t}))}{\sum_{\mathrm{i}=1}^{\mathrm{n}} \mathrm{w}_{\mathrm{i}}(\mathrm{Z}(\mathrm{t}))} ; \mathrm{w}_{\mathrm{i}}(\mathrm{Z}(\mathrm{t}))=\prod_{\mathrm{j}=1}^{\mathrm{q}} \mathrm{M}_{\mathrm{ji}}(\mathrm{Z}(\mathrm{t})) .
\end{aligned}
$$

The approach proposed in this research project is based on the quadratic Lyapunov function. Choosing a Lyapunov function that guarantee stability in simple terms requires a symmetric matrix which is positively defined. Practically, it is simple to implement using $V(x(k))$ such as:

$$
\mathrm{V}(\mathrm{x}(\mathrm{k}))=\mathrm{X}_{\mathrm{k}}^{\mathrm{T}} \mathrm{PX}_{\mathrm{k}}
$$

The Discrete T-S fuzzy system is asymptotically stable. If a common matrix $P=P^{T}>0$ exists, then the following LMI is feasible (Boyd et al., 1994, Tanaka et al., 1998):

$$
\left\{\begin{array}{c}
\mathrm{P}>0 \\
\mathrm{~A}_{\mathrm{i}}^{\mathrm{T}} \mathrm{PA}_{\mathrm{i}}-\mathrm{P}<0 .
\end{array}\right.
$$

\subsection{Stabilization of a discret multiple state feedback}

The stabilization technique used in this paper is the Parallel Distributed Control (PDC) law, which is written as follow:

$$
\mathrm{u}(\mathrm{k})=-\sum_{\mathrm{i}=1}^{\mathrm{n}} \mu_{\mathrm{i}}(\mathrm{Z}(\mathrm{k})) \mathrm{K}_{\mathrm{i}} \mathrm{x}(\mathrm{k})
$$

Using the discrete multiple models previously described in (8), a closed loop control law by PDC is given by:

$$
\left\{\begin{array}{c}
\mathrm{x}(\mathrm{k}+1)=\sum_{\mathrm{i}=1}^{\mathrm{n}} \sum_{\mathrm{j}=1}^{\mathrm{n}} \mu_{\mathrm{i}}(\mathrm{Z}(\mathrm{k})) \mu_{\mathrm{j}}(\mathrm{Z}(\mathrm{k})) \delta_{\mathrm{ij}} \mathrm{x}(\mathrm{k}) . \\
\delta_{\mathrm{ij}}=\mathrm{A}_{\mathrm{i}}-\mathrm{B}_{\mathrm{i}} \mathrm{K}_{\mathrm{j}} .
\end{array}\right.
$$


Hypothesis: The two matrices $\left(A_{i}, B_{i}\right)$ are assumed to be controllable. It is said that the multiple models is locally controllable as described in (Euntai and Heejin, 2000).

The stabilization conditions for discrete-time multiple models using closed-loop PDC control law require a symmetric matrix $P>0$ and matrices $K_{i}, \forall i \in I_{n}$ such as:

$$
\begin{gathered}
\left\{\begin{array}{c}
\mathrm{l}_{\mathrm{d}}\left(\delta_{\mathrm{ii}}, \mathrm{P}\right)<0, \forall \mathrm{i} \in \mathrm{I}_{\mathrm{n}}, \\
\mathrm{l}_{\mathrm{d}}\left(\delta_{\mathrm{ij}}, \mathrm{P}\right) \leq 0, \forall \mathrm{i}, \mathrm{j} \in \mathrm{I}_{\mathrm{n}}^{2} \text { and }, \\
\mu_{\mathrm{i}}(\mathrm{Z}(\mathrm{k})) \mu_{\mathrm{j}}(\mathrm{Z}(\mathrm{k})) \neq 0 .
\end{array}\right. \\
\mathrm{l}_{\mathrm{d}}\left(\delta_{\mathrm{ij}}, \mathrm{P}\right)=\left[\frac{\delta_{\mathrm{ij}}+\delta_{\mathrm{ji}}}{2}\right]^{\mathrm{T}} \mathrm{P}\left[\frac{\delta_{\mathrm{ij}}+\delta_{\mathrm{ji}}}{2}\right]-\mathrm{P}, \text { with } \\
\delta_{\mathrm{ij}}=\mathrm{A}_{\mathrm{i}}-\mathrm{B}_{\mathrm{i}} \mathrm{K}_{\mathrm{j}} .
\end{gathered}
$$

The following conditions are:

$$
\left(A_{i}-B_{i} K_{i}\right)^{T} P\left(A_{i}-B_{i} K_{i}\right)-P<0 .
$$

In pre and post multiplying by $P^{-1}$, we obtain:

$$
\mathrm{P}^{-1}\left(\mathrm{~A}_{\mathrm{i}} \mathrm{P}^{-1}-\mathrm{B}_{\mathrm{i}} \mathrm{K}_{\mathrm{i}} \mathrm{P}^{-1}\right)^{\mathrm{T}} \mathrm{P}\left(\mathrm{A}_{\mathrm{i}} \mathrm{P}^{-1}-\mathrm{B}_{\mathrm{i}} \mathrm{K}_{\mathrm{i}} \mathrm{P}^{-1}\right)>0 .
$$

It's assumed that $X=P^{-1}$ and $N_{i}=K_{i} P^{-1}$; then we obtain:

$$
X\left(A_{i} X-B_{i} K_{i} X\right)^{T} P\left(A_{i} X-B_{i} N_{i}\right)>0 .
$$

Using the Schur complement, the above condition is written in the form of LMI:

$$
\left[\begin{array}{cc}
\mathrm{X} & * \\
\mathrm{~A}_{\mathrm{i}} \mathrm{X}-\mathrm{B}_{\mathrm{i}} \mathrm{N}_{\mathrm{i}} & \mathrm{X}
\end{array}\right]>0 \forall i \in I_{n}
$$

By using the same method, the condition $l_{d}\left(\delta_{i j}, P\right) \leq 0$ is written as:

$$
\left[\begin{array}{cc}
\mathrm{X} & * \\
\frac{\mathrm{A}_{\mathrm{i}}+\mathrm{A}_{\mathrm{j}}}{2} \mathrm{X}-\frac{1}{2}\left(\mathrm{~B}_{\mathrm{j}} \mathrm{N}_{\mathrm{i}}+\mathrm{B}_{\mathrm{i}} \mathrm{N}_{\mathrm{j}}\right) & \mathrm{X}
\end{array}\right] \geq 0 \forall(i, j) \in I_{n}^{2}, i<j .
$$

Now the discrete multiple models in (8) is globally asymptotically stable via improved PDC control law, especially if there are symmetric matrices such as $P=P^{T}>0$ and $Q=Q^{T} \geq 0$ which verifies (Chadli et al., 2002):

$$
\begin{gathered}
\left\{\begin{array}{c}
\mathrm{l}_{\mathrm{d}}\left(\delta_{\mathrm{ii}}, \mathrm{P}\right)+(\mathrm{r}-1) \mathrm{Q}<0, \forall \mathrm{i} \in \mathrm{I}_{\mathrm{n}}, \\
\mathrm{l}_{\mathrm{d}}\left(\delta_{\mathrm{ij}}, \mathrm{P}\right)-\mathrm{Q} \leq 0, \forall \mathrm{i}, \mathrm{j} \in \mathrm{I}_{\mathrm{n}}^{2}, \mathrm{i}<\mathrm{j}, \\
\mu_{\mathrm{i}}(\mathrm{Z}(\mathrm{k})) \mu_{\mathrm{j}}(\mathrm{Z}(\mathrm{k})) \neq 0 .
\end{array}\right. \\
\mathrm{l}_{\mathrm{d}}\left(\delta_{\mathrm{ij}}, \mathrm{P}\right)=\left[\frac{\delta_{\mathrm{ij}}+\delta_{\mathrm{ji}}}{2}\right]^{\mathrm{T}} \mathrm{P}\left[\frac{\delta_{\mathrm{ij}}+\delta_{\mathrm{ji}}}{2}\right]-\mathrm{P} .
\end{gathered}
$$


By using matrices $Q_{i j}, Q_{i j}$, due to the conservatism and pessimism results, we can define the following theorem presented in (Euntai and Heejin, 2000). Theorem: if matrices $P=P^{T}>0, Q_{i j}=Q_{i j}^{T}$ and matrices $K_{i}$ exists, which verify the equation below:

$$
\begin{gathered}
\left\{\begin{array}{c}
\mathrm{l}_{\mathrm{d}}\left(\delta_{\mathrm{ii}}, \mathrm{P}\right)+(\mathrm{r}-1) \mathrm{Q}<0, \forall \mathrm{i} \in \mathrm{I}_{\mathrm{n}} . \\
\mathrm{l}_{\mathrm{d}}\left(\delta_{\mathrm{ij}}, \mathrm{P}\right)-\mathrm{Q} \leq 0, \forall \mathrm{i}, \mathrm{j} \in \mathrm{I}_{\mathrm{n}}^{2}, \mathrm{i}<\mathrm{j} . \\
\mu_{\mathrm{i}}(\mathrm{Z}(\mathrm{k})) \mu_{\mathrm{j}}(\mathrm{Z}(\mathrm{k})) \neq 0 .
\end{array}\right. \\
\mathrm{Q}=\left[\begin{array}{ll}
\mathrm{Q}_{11} \ldots . & \mathrm{Q}_{1 \mathrm{n}} \\
\mathrm{Q}_{1 \mathrm{n}} \ldots . & \mathrm{Q}_{\mathrm{nn}}
\end{array}\right] .
\end{gathered}
$$

Then the multiple models in (8) is globally asymptotically stable. Thus, the BMIs of the previous theorem can be transformed into LMIs, which gives:

$$
\left\{\begin{array}{c}
X=P^{-1} . \\
Y_{i i}=X Q_{i j} X . \\
K_{i}=N_{i} X^{-1} . \\
\forall i \in I_{n} .
\end{array}\right.
$$

\section{Synthesis of multi-observers}

Synthesis techniques based on state feedback (PDC control law) need to have the entire state vector $x(k)$, as shown in equations (12)-(13). Therefore, we need to rebuild the state based system using the following multiobservers:

$$
\left\{\begin{array}{c}
\hat{x}(k+1)=\mu_{i}(Z(k))\left(A_{i} \hat{x}(k)+B_{i} u(k)\right)+L_{i}(y(k)-\hat{y}(k)) . \\
\hat{y}(k)=\sum_{i=1}^{n} \mu_{i}(Z(k)) C_{i} \hat{x}(k) .
\end{array}\right.
$$

Knowing that the estimation of the state vector error is given by:

$$
\tilde{\mathrm{x}}(\mathrm{k})=\mathrm{x}(\mathrm{k})-\hat{\mathrm{x}}(\mathrm{k}) .
$$

The dynamics of the estimation error could then be written as:

$$
\left\{\begin{array}{c}
\hat{\mathrm{x}}(\mathrm{k}+1)=\sum_{\mathrm{i}=1}^{\mathrm{n}} \sum_{\mathrm{j}=1}^{\mathrm{n}} \mu_{\mathrm{i}}(\mathrm{Z}(\mathrm{k})) \mu_{\mathrm{j}}(\mathrm{Z}(\mathrm{k})) \theta_{\mathrm{ij}} \tilde{\mathrm{x}}(\mathrm{k}) \\
\theta_{\mathrm{ij}}=\mathrm{A}_{\mathrm{i}}-\mathrm{L}_{\mathrm{i}} \mathrm{C}_{\mathrm{j}}, \forall(\mathrm{i}, \mathrm{j}) \in \mathrm{I}_{\mathrm{n}}^{2}
\end{array}\right.
$$

The synthesis of a multiple observer is used in determining local gains $L_{i} \in I_{n}$ to ensure the convergence to zero of the dynamics of the estimation error. We have to ensure that there is $P=P^{T}>0$ and matrices $L_{i} \in I_{n}$ that verify:

$$
\begin{gathered}
\left\{\begin{array}{c}
l_{d}\left(\theta_{i i}, P\right)<0, \forall i \in I_{n} . \\
l_{d}\left(\theta_{i j}, P\right) \leq 0, \forall i, j \in I_{n}^{2} . \\
\mu_{i}(Z(k)) \mu_{j}(Z(k)) \neq 0 .
\end{array}\right. \\
l_{d}\left(\theta_{i j}, P\right)=\left[\frac{\theta_{i j}+\theta_{j i}}{2}\right]^{T} P\left[\frac{\theta_{i j}+\theta_{j i}}{2}\right]-P .
\end{gathered}
$$

Then the estimation error converges to zero. The conditions of the previous theorem can be transformed into LMIs using the Schur complement: 


$$
\begin{gathered}
{\left[\begin{array}{cr}
P \frac{A_{i}+A_{j}}{2}-\frac{1}{2}\left(N_{j} C_{j}+N_{j} C_{i}\right) & P
\end{array}\right] \geq 0 .} \\
\forall(i, j) \in I_{n}^{2}, \mathrm{i}<j .
\end{gathered}
$$

The previous multiple observers may be improved by the following theorem. The multiple observers is globally asymptotically stable if there exist a symmetric matrices $P>0, Q_{i j}$ and $L_{i} \in I_{n}$ that satisfy:

$$
\left\{\begin{array}{c}
\mathrm{l}_{\mathrm{d}}\left(\theta_{\mathrm{ii}}, P\right)+\mathrm{Q}_{\mathrm{ii}}<0, \forall \mathrm{i} \in \mathrm{I}_{\mathrm{n}} . \\
\mathrm{l}_{\mathrm{d}}\left(\theta_{\mathrm{ij}}, \mathrm{P}\right)+\mathrm{Q}_{\mathrm{ij}} \leq 0, \forall \mathrm{i}, \mathrm{j} \in \mathrm{I}_{\mathrm{n}}^{2} . \\
{\left[\begin{array}{cc}
\mathrm{Q}_{11} & \mathrm{Q}_{1 \mathrm{n}} \\
\mathrm{Q}_{1 \mathrm{n}} & \mathrm{Q}_{\mathrm{nn}}
\end{array}\right] .} \\
\mu_{\mathrm{i}}(\mathrm{z}(\mathrm{k})) \mu_{\mathrm{j}}(\mathrm{z}(\mathrm{k})) \neq 0 . \\
\theta_{\mathrm{ij}}=\mathrm{A}_{\mathrm{i}}-\mathrm{L}_{\mathrm{i}} \mathrm{C}_{\mathrm{j}} \forall(\mathrm{i}, \mathrm{j}) \in \mathrm{I}_{\mathrm{n}}^{2} .
\end{array}\right.
$$

The matrix inequalities are written as:

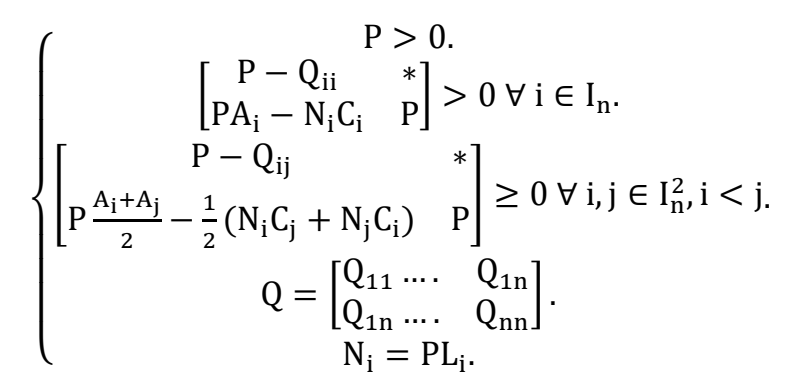

\section{Numerical example}

In this section, we apply our theoretical development to confirm and explain our contribution with a numerical example.

\subsection{System identification using N4SID1 and 2}

Here, we considered the following described by the state matrix A, input vector B, output matrix C, and the direct transmission matrix D.

These matrices are in continuous time and, however, we must discretize them so they can be usable in our algorithms. For this purpose, the following N4SID representation was considered:

$$
\begin{gathered}
x_{k+1}=A x_{k}+B u_{k}+w_{k} . \\
y_{k}=C x_{k}+D u_{k}+v_{k} .
\end{gathered}
$$

We will identify a multivariable system, which has two inputs and two outputs. First, we will show the estimation results of N4SID1 and then N4SID2. The identification results are obtained for a sampling time $\mathrm{Te}=0.1$ second. In addition, we used the pseudo-random binary signal (PRBS), shown in Figure 4 , in order to add noise in the data and demonstrate algorithm robustness. 


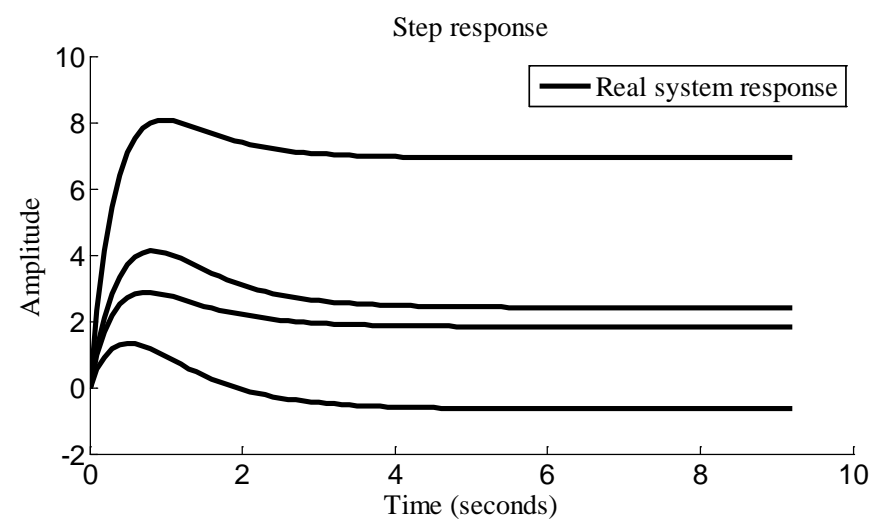

Figure 3. Real continuous system step response.

The studied system is a multiple-input / multiple-output (MIMO) system, as the inputs are u1 and $\mathrm{u} 2$ and the outputs are y1 and y2. We excite the system by an input step and we have then, as shown in Figure 3, different combinations of inputs / outputs. Thereafter, we simulated using Matlab environment the tracing of Bode magnitude plot associated to the MIMO system, which is described by a state representation formed by the A, B, C, and D matrix.

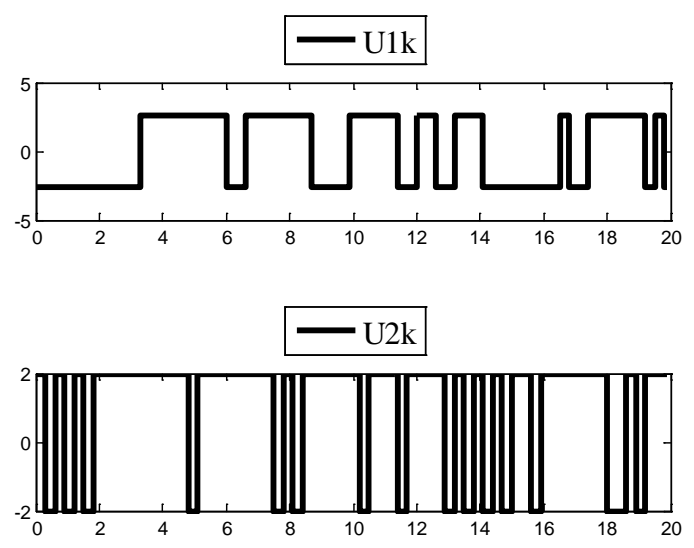

Figure 4. Generation of two PRBS for identification of 2 input system.

A pseudo-random binary sequence "PRBS" is an exciter signal that correctly identifies the parameters of a dynamic model like a black box model. Figure 4 shows the two PRBS used for the system identification. The first PRBS have a [2.6, -2.6] amplitude called U1k, while the second PRBS signal have a [2. 2] amplitude called U2k. The binary pseudorandom sequence is shown between samples [1, 200], knowing that the sampling time is equal to 0.1. The PRBS is a train of amplitude niche (variants between two terminals) that is approached from a discrete white noise rich in frequency.

Figure 6 represents the frequency responses, where we note a similarity between the results with different combinations of inputs / outputs which confirm the identification effectiveness of N4SID algorithm. Therefore, Figure 6 shows the comparison between the real discretized system and N4SID 2 algorithms. 


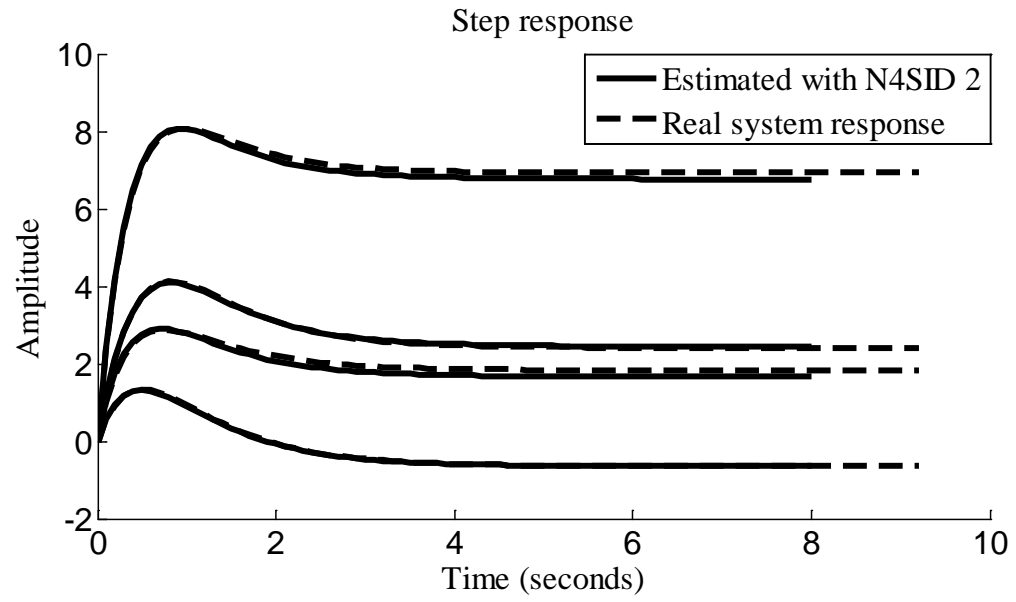

Figure 5. Step response using N4SID2. 

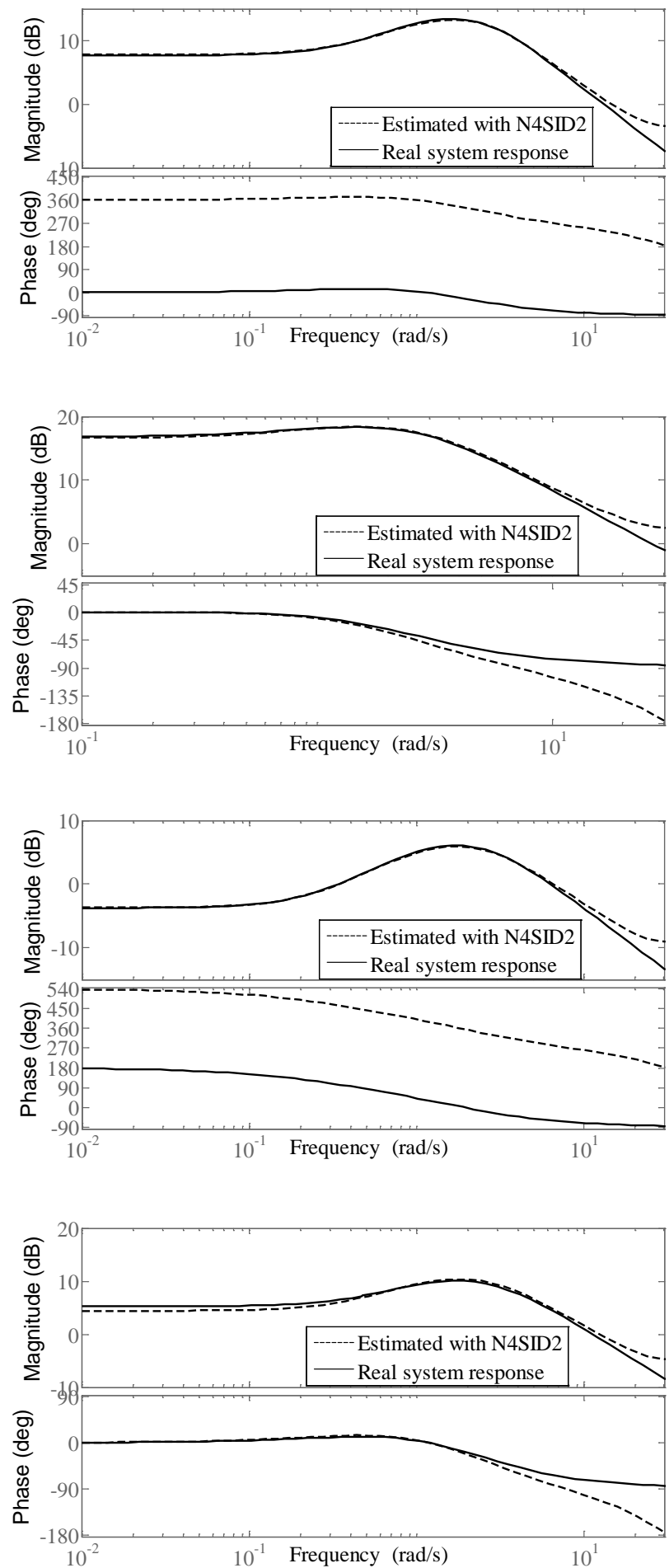

Figure 6. Frequency response using N4SID2 algorithm. 


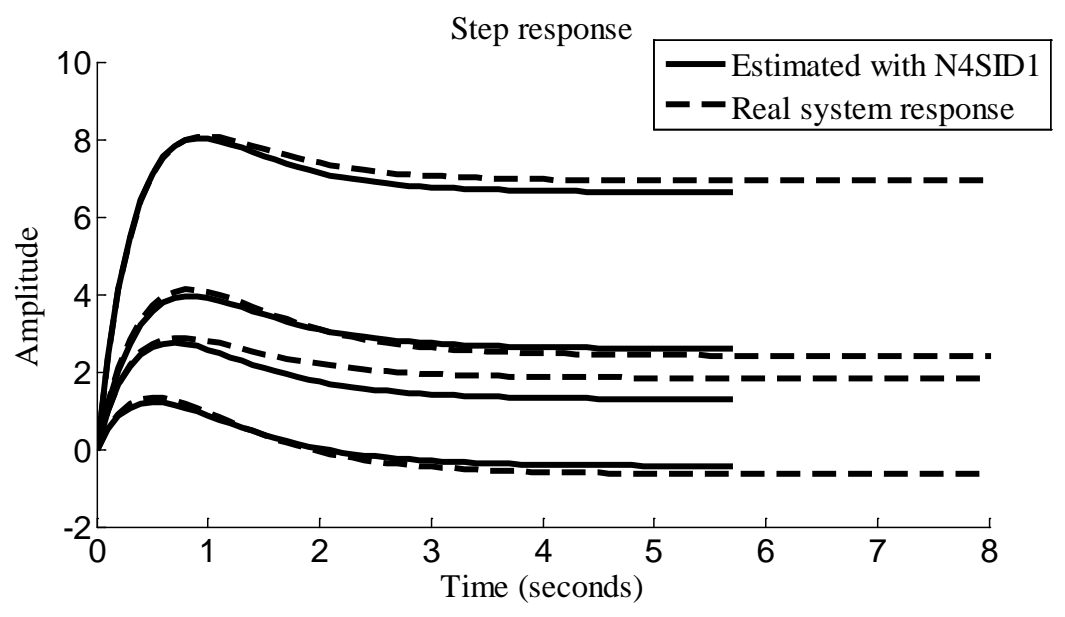

Figure 7. Step response using N4SID1 algorithm.

Figure 7 represents the step responses of the discretised real system and the identified system with N4SID 1. They are presented respectively by the black continued line and the black discontinuous line. Computation time is an important parameter for industrial process. Therefore, Table 1 compare the identification time for the MIMO system. Also, Table 2 shows the mean squared error (MSE) between the estimated models by both N4SID algorithms and MOESP algorithm. The lowest value indicates the best algorithm which is N4SID2.

Table 1. Algorithm computation time for identification of one MIMO system.

\begin{tabular}{|lll|}
\hline N4SID 1 & N4SID 2 & MOESP \\
\hline $\mathbf{0 . 2 9 6 9}$ & 0.5625 & 0.6250 \\
\hline
\end{tabular}

Table 2. MSE in time domain for both N4SID and MOESP algorithms compared to the real system.

\begin{tabular}{|lll|}
\hline N4SID 1 & N4SID 2 & MOESP \\
\hline $\mathbf{2 . 4 9 4 0}$ & 0.5625 & 0.6250 \\
\hline
\end{tabular}




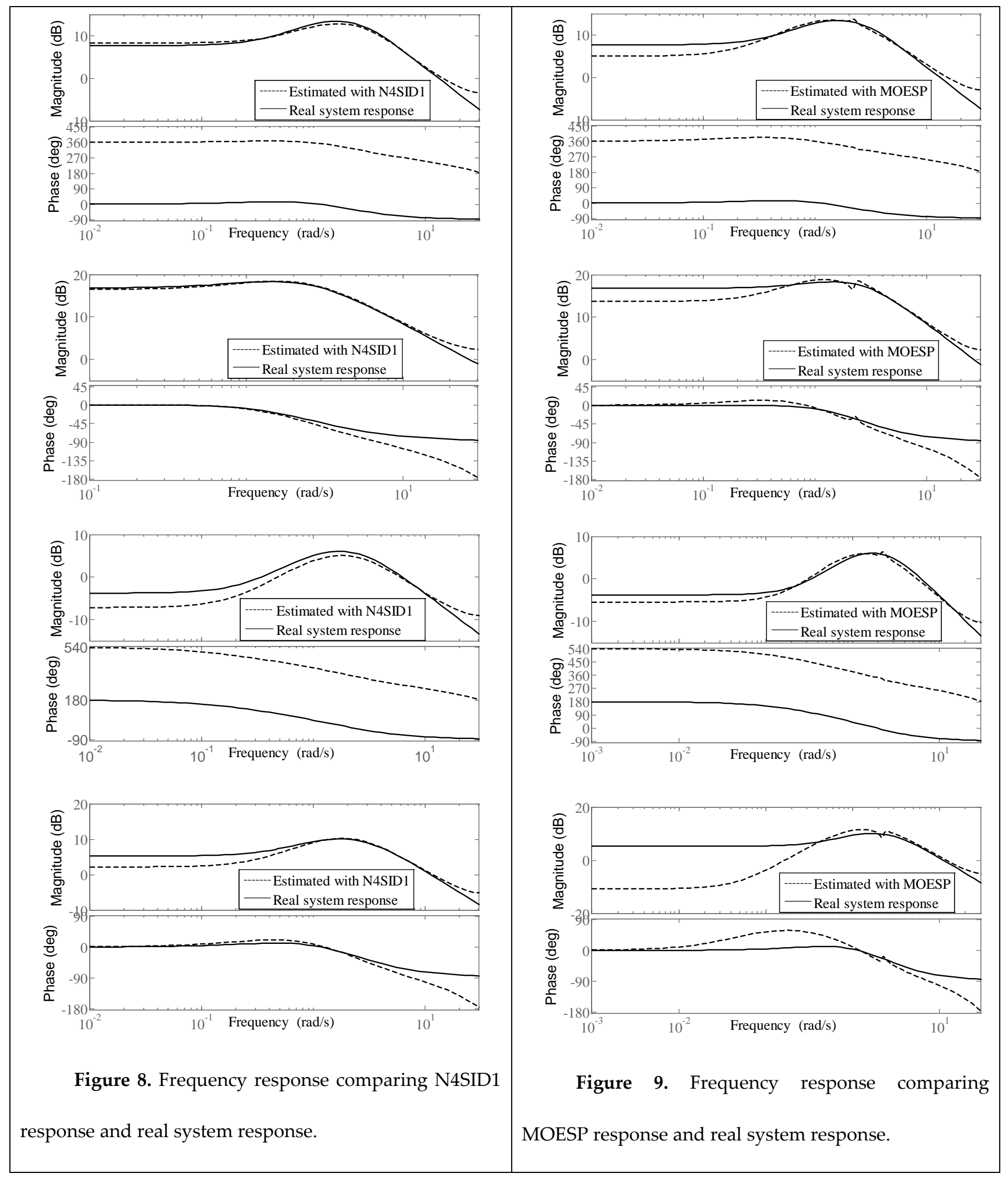



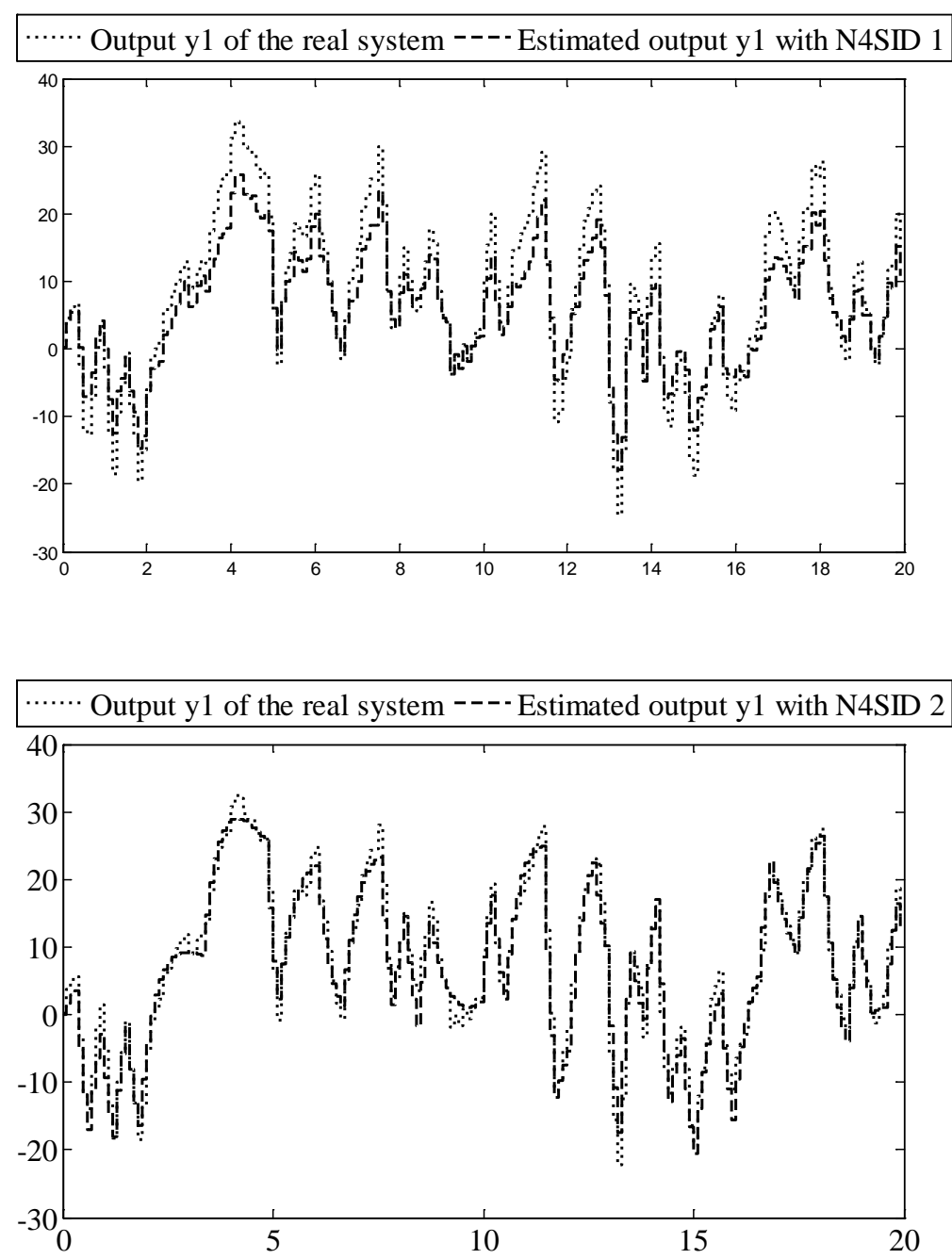

Figure 10. Zoom on first results of samples [1,200] using N4SID1 and N4SID2.

Figure 10 represents the comparison between the outputs of the discretised real model and the identified outputs model. It makes comparisons between the simulations of the obtained model with measured data, in order to validate a model.

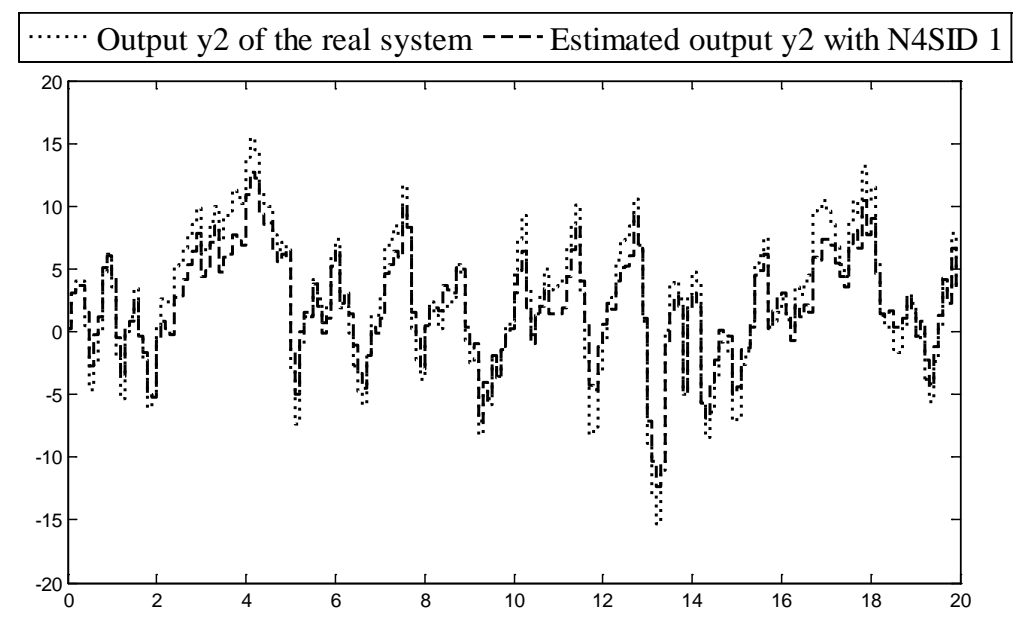




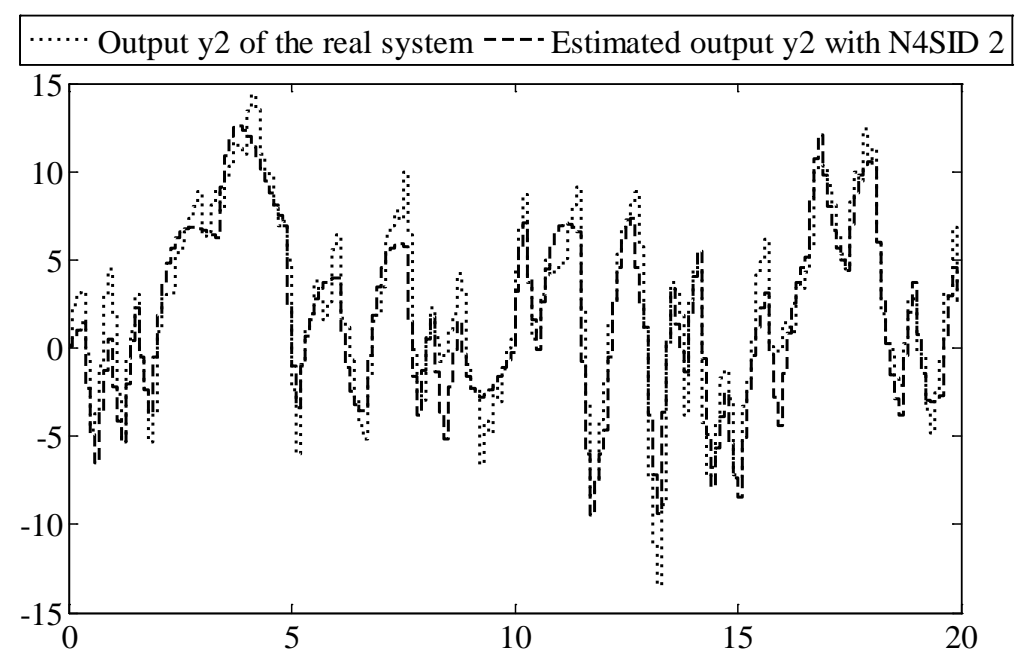

Figure 11. Zoom on second output results of samples [1,200] using N4SID1 and N4SID2.

Figure 11 represents the comparison between the outputs y2 of the discretized real model and the output y2 of the estimated models with the two identification algorithms N4SID 1 and N4SID 2.

\subsection{Multiple model stabilization}

In this part of the article, we are interested in stabilization of the multiple models. In the previous section, we studied only one model with two inputs and two outputs. In this section, we study the stability and stabilization of four MIMO models (each model are LTI with two inputs and two outputs). The T-S is formed by the matrices identified by N4SID2 since the results are more accurate than N4SID1 and MOESP using MSE criterion (see Table 2). The states matrices Ai, represented in equation (8), that represent the multiple models, the input vectors, and the output vectors of each local linear model are given as:

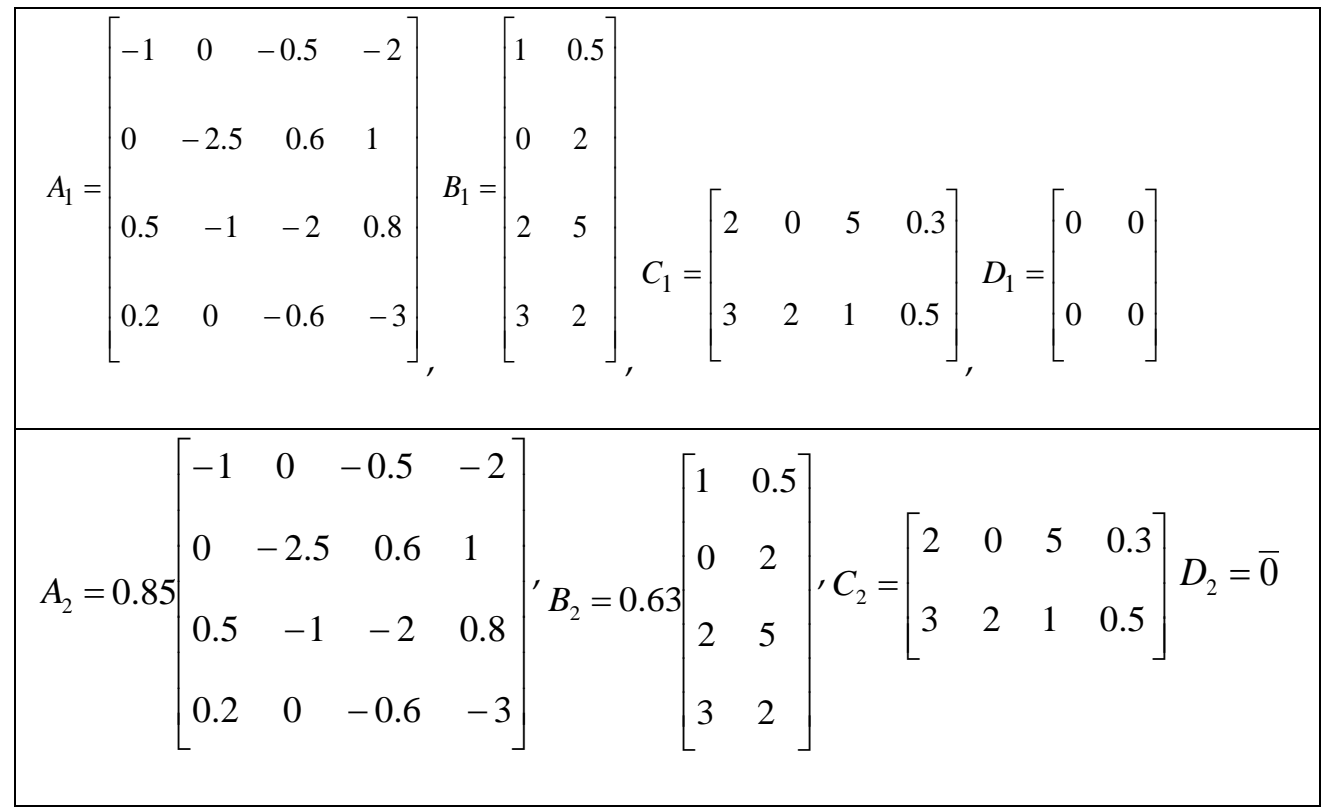




$$
\begin{aligned}
& A_{3}=0.8\left[\begin{array}{cccc}
-1 & 0 & -0.5 & -2 \\
0 & -2.5 & 0.6 & 1 \\
0.5 & -1 & -2 & 0.8 \\
0.2 & 0 & -0.6 & -3
\end{array}\right], B_{3}=0.6\left[\begin{array}{ll}
1 & 0.5 \\
0 & 2 \\
2 & 5 \\
3 & 2
\end{array}\right], C_{3}=\left[\begin{array}{llll}
2 & 0 & 5 & 0.3 \\
3 & 2 & 1 & 0.5
\end{array}\right], D_{3}=\left[\begin{array}{ll}
0 & 0 \\
0 & 0
\end{array}\right] \\
& A_{4}=0.6\left[\begin{array}{llll}
-1 & 0 & -0.5 & -2 \\
0 & -2.5 & 0.6 & 1 \\
0.5 & -1 & -2 & 0.8 \\
0.2 & 0 & -0.6 & -3
\end{array}\right], B_{4}=0.4\left[\begin{array}{ll}
1 & 0.5 \\
0 & 2 \\
2 & 5 \\
3 & 2
\end{array}\right], C_{4}=\left[\begin{array}{llll}
2 & 0 & 5 & 0.3 \\
3 & 2 & 1 & 0.5
\end{array}\right], D_{4}=\overline{0}
\end{aligned}
$$

From (26) and (34), the feedback gain $K_{i}$, the matrix $P$, and the gain $L_{i}$ matrix can be obtained as:

$$
\left\{\begin{array}{l}
P=X^{-1} . \\
K_{i}=N_{i} P . \\
N_{i}=P L_{i} .
\end{array}\right.
$$

Solving LMIs system using (34) and (37), we obtain:

$$
\begin{aligned}
& P=\left[\begin{array}{cccc}
0.6384 & -0.1033 & 0.0320 & -0.0867 \\
-0.1033 & 0.1409 & -0.0300 & -0.0324 \\
0.0320 & -0.0300 & 0.1888 & 0.0465 \\
-0.0867 & -0.0324 & 0.0465 & 0.6214
\end{array}\right], K 1=\left[\begin{array}{cccc}
-0.1654 & 0.3336 & -0.2190 & -2.2542 \\
-0.3941 & -0.1941 & -0.0100 & 1.1506
\end{array}\right], \\
& K 2=\left[\begin{array}{cccc}
-0.1609 & 0.2250 & -0.0541 & -1.5345 \\
-0.5701 & -0.0491 & -0.1422 & 0.7680
\end{array}\right], K 3=\left[\begin{array}{cccc}
-0.1374 & 0.2276 & -0.1572 & -1.6386 \\
-0.5965 & -0.0446 & -0.0513 & 0.8109
\end{array}\right], \\
& K 4=\left[\begin{array}{rrrr}
-0.1687 & 0.4688 & -0.1928 & -2.0304 \\
-0.7914 & -0.1578 & -0.1185 & 1.0189
\end{array}\right] \\
& L 1=\left[\begin{array}{ll}
-0.2415 & -0.6126 \\
0.6019 & -1.5255 \\
-0.1902 & 0.2630 \\
0.1673 & -0.2075
\end{array}\right], L 2=\left[\begin{array}{ll}
-0.4337 & -0.4415 \\
1.2732 & -2.9498 \\
-0.3946 & 0.6706 \\
0.3345 & -0.5000
\end{array}\right], L 3=\left[\begin{array}{ll}
-0.3757 & -0.5576 \\
1.3848 & -3.1157 \\
-0.3425 & 0.6031 \\
0.3256 & -0.4825
\end{array}\right], L 4=\left[\begin{array}{ll}
-0.5692 & -0.4968 \\
0.7727 & -1.6519 \\
-0.2834 & 0.2843 \\
0.1315 & -0.1060
\end{array}\right] .
\end{aligned}
$$

Figure 12 shows respectively the output $y 1(t)$ and $y 2(t)$ of the multivariable fuzzy system with real and identified parameters. The system is globally asymptotically stable because we observed that $\mathrm{y} 1(\mathrm{t})$ and $\mathrm{y} 2(\mathrm{t})$ converge towards zero. 

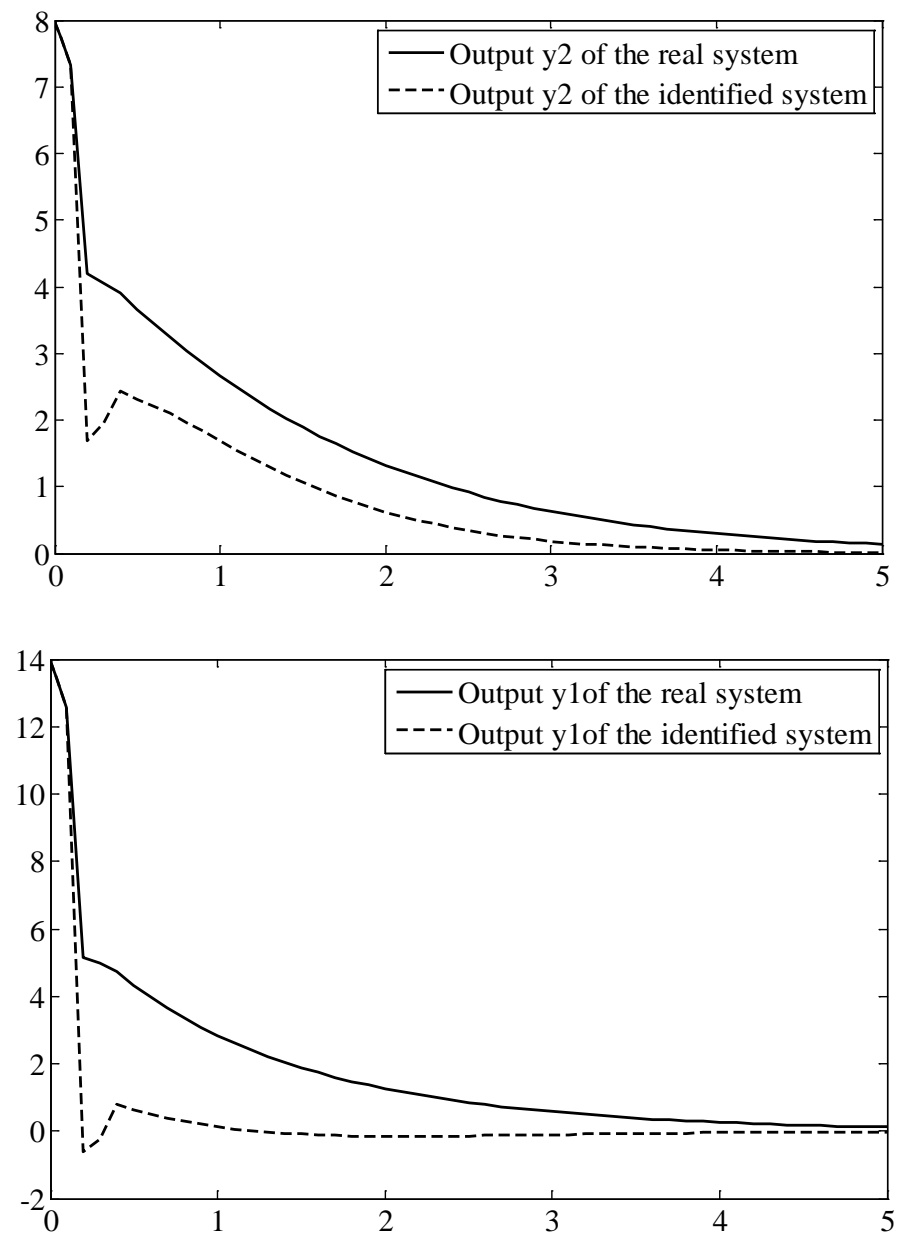

Figure 12. Output $y 1, y 2$ of the real and identified system stabilized with the improved PDC control law.

However, these results present the stabilization of the multiple model system. The real system and the identified one described by Figure 12 were observed as a global asymptotic stabilization. The stabilization of the identified multiple model built it by the estimated matrices through N4SID 2 algorithm. We observe also that all trajectories of the above fuzzy system converge to zero.

\section{Conclusion}

In this paper, we used two N4SID algorithms for the identification of multivariable systems and we compare our proposed algorithm with the MOESP. The N4SID algorithms subspace identification methods differ from other classic identification algorithms in their concept, interpretation, and computational implementation. We demonstrate the efficiency of an improved N4SID algorithm. In the second section of this paper, we used the LTI identified model with N4SID2 to build the T-S nonlinear system. After that, we studied various conditions for the global asymptotic stability system. This approach ensures the global asymptotic stability of the closed loop T-S fuzzy systems. Therefore, the stabilization results require the quadratic Lyapunov function and all components of the state vector. For this reason, we constructed a multiobserver to estimate the state vector. 


\section{Acknowledgments}

This work is supported financially by the Natural Sciences and Engineering Research Council of Canada (NSERC) under the grant number EGP 477206 - 14, held by Martin Otis.

\section{Appendix A: Mathematical tools}

In this appendix, we present the mathematical tools of linear algebra used in subspace method. We define first the extended observability matrix (we extend the observability to an order that is superior to the one of the system) (Van Overschee and De Moor, 1994):

$$
\Gamma_{i}=\left(\begin{array}{l}
C \\
C A \\
\cdots \\
C A^{i-1}
\end{array}\right) .
$$

Then, the reversed extended controllability matrix is:

$$
\Delta_{i}=\left(\begin{array}{llll}
A^{i-1} B & A^{i-2} B \quad \ldots B
\end{array}\right) .
$$

The input block Hankel matrix is defined as:

$$
U_{0 \mid i-1}=\left(\begin{array}{ccccc}
u_{0} & u_{1} & u_{2} & \ldots & u_{j-i} \\
u_{1} & u_{2} & u_{3} & \ldots & u_{j} \\
\ldots & \ldots & \ldots & \ldots & \ldots \\
u_{i-1} & u_{i} & u_{i+1} & \ldots & u_{i+j-2}
\end{array}\right) .
$$

and the output block Hankel matrix is:

$$
Y_{0 \mid i-1}=\left(\begin{array}{ccccc}
y_{0} & y_{1} & y_{2} & \cdots & y_{j-i} \\
y_{1} & y_{2} & y_{3} & \cdots & y_{j} \\
\cdots & \cdots & \cdots & \cdots & \cdots \\
y_{i-1} & y_{i} & y_{i+1} & \cdots & y_{i+j-2}
\end{array}\right) .
$$

The following matrices are used: $U_{p}=U_{0 \mid i-1}, \quad U_{f}=U_{i \mid 2 i-1}, U_{p}^{+}=U_{0 \mid i}$ and $U_{f}^{-}=$ $U_{i+1 \mid 2 i-1}$. A similar definitions is presented for Hankel block output matrices: $Y_{p}, Y_{p}^{+}, Y_{f}$ and, $Y_{f}^{+}$where subscript stands ' $\mathrm{f}$ ' for the "future" and ' $\mathrm{P}$ ' for the "past".

For the stochastic sub- model (6)-(7), we define:

$$
\left\{\begin{array}{l}
P^{s}=E\left[x_{k}^{s}\left(x_{k}^{s}\right)^{T}\right]=A P^{s} A^{T}+Q^{s} . \\
\nabla=E\left[x_{k+1}^{s}\left(y_{k}^{s}\right)^{T}\right]=A P^{s} C^{T}+S^{s} . \\
\Lambda_{0}=E\left[x_{k+1}^{s}\left(y_{k}^{s}\right)^{T}\right]=C P^{S} C^{T}+R^{s} .
\end{array}\right.
$$

The matrix $\Delta_{i}^{S}$ associated with the stochastic sub-model is: 


$$
\Delta_{i}^{s}=\left(\begin{array}{llll}
A^{i-1} \nabla & \ldots & A \nabla & \nabla
\end{array}\right) .
$$

The Block lower triangular Toeplitz matrix and the block Toeplitz cross covariance matrix are:

$$
\begin{aligned}
H_{i}^{d} & =\left(\begin{array}{ccccc}
D & 0 & 0 & \ldots & 0 \\
C B & D & 0 & \ldots & 0 \\
C A B & C B & D & \ldots & 0 \\
\ldots & \ldots & \ldots & \ldots & \ldots \\
C A^{i-2} B & C A^{i-3} B & C A^{i-4} B & \ldots & D
\end{array}\right) . \text { and } \\
H_{i}^{s} & =\left(\begin{array}{ccccc}
I & 0 & 0 & \ldots & 0 \\
C K & I & 0 & \ldots & 0 \\
C A K & C K & I & \ldots & 0 \\
\ldots & \ldots & \ldots & \ldots & \ldots \\
C A^{i-2} K & C A^{i-3} K & C A^{i-4} K & \ldots & K
\end{array}\right) .
\end{aligned}
$$

\section{Appendix B: Kalman states}

From the matrices of block Hankel, the matrices system are determined directly through the determination of projections from which to estimate the two states sequences which are the outputs of the extended Kalman filter (Van Overschee and De Moor, 1994):

$$
\begin{aligned}
& X_{i}^{d}=\left[x_{i}^{d} \cdots x_{i+j-1}^{d}\right] . \\
& X_{i}^{s}=\left[x_{i}^{s} \cdots x_{i+j-1}^{s}\right] .
\end{aligned}
$$

If both noises are jointly Gaussian, we have the best estimates of the Kalman states in the quadratic mean sense. If we knew the initial states $\hat{X}_{0}$ and its covariance $P_{0}$, inputs and outputs $u=$ $\left[u_{1} u_{2} \cdots u_{n}\right], y=\left[\begin{array}{llll}y_{1} & y_{2} & \cdots & y_{n}\end{array}\right]$ and all system matrices $A, B, C, D, Q, S, R$, the states can be easily computed using the following recursive formulas:

$$
\left\{\begin{array}{l}
\hat{x}_{k}=A \hat{x}_{k-1}+B u_{k-1}+K_{k-1}\left(y_{k-1}-C \hat{x}_{k-1}-D u_{k-1}\right) . \\
K_{k-1}=\left(A P_{k-1} C^{T}+\nabla\right)\left(\Lambda_{0}+C P_{k-1} C^{T}\right)^{-1}\left(A P_{k-1} C^{T}+\nabla\right)^{T} . \\
P_{k}=A P_{k-1} A^{T}-\left(A P_{k-1} C^{T}+\nabla\right)\left(\Lambda_{0}+C P_{k-1} C^{T}\right)^{-1}\left(A P_{k-1} C^{T}+\nabla\right)^{T} .
\end{array}\right.
$$

Based on last equation, we computed the Kalman gain and recursively defined the error covariance matrix $E\left[\left(x_{k}-\hat{x}_{k}\right)\left(x_{k}-\hat{x}_{k}\right)^{T}\right]$ which estimates $P^{s}$.

From previous equations, it was possible to establish the following formulas:

$$
\begin{aligned}
& \hat{X}_{i+1}=A \hat{X}_{i}+B U_{i \mid i}+K_{i}\left(Y_{i \mid i}-C \hat{X}_{i}-D U_{i \mid i}\right) . \\
& Y_{i \mid i}=C \hat{X}_{i}+B U_{i \mid i}+\left(Y_{i \mid i}-C \hat{X}_{i}-D U_{i \mid i}\right)
\end{aligned}
$$


If it was possible to obtain Kalman states directly from input- output data, the equations above would satisfy the assumption on consistent applications of least squares to estimate system matrices $A, B, C, D$. We determine from the residuals matrices the covariance matrices $\left(Q^{S}, S^{S}, R^{S}\right)$.

\section{Appendix C: Orthogonal projection}

In this appendix, we present the geometric tools of linear algebra used in subspace method. We assume that the matrices $A, B, C$ are given.

The orthogonal projection of the row space of $A$ into the row space of $B$ is defined as (Van Overschee and De Moor, 1994):

$A / B=A \Pi_{B}=A B\left(B B^{t}\right)^{+} B$.

Where $(.)^{+}$denotes the Moore-Penrose of the matrix (.) and $\Pi_{B}=B^{T}\left(B \cdot B^{T}\right)^{+} . B$. It is important to note that $\Pi_{B}$ defined the operator of orthogonal projection that projects the row space of matrix onto the row space of the matrix $B$.

The projection of the row space of $A$ into the orthogonal complement of the row space of $B$ is:

$$
A / B^{\perp}=A \Pi_{B^{\perp}}=A-A / B=A\left(I_{j}-\Pi_{B}\right),
$$

Where $B^{\perp}$ denotes a base of the orthogonal space to the row space of $B$, and $\prod_{B^{\perp}}$ defines the geometric operator that projects the row space of a matrix onto the orthogonal complement to the row space of the matrix B. From the expression of $\Pi_{B}$ and $\Pi_{B^{\perp}}$, one can easily show that matrix $A$ can be decomposed into two matrices where their row spaces are orthogonal such as: $A=A \Pi_{B}+A \Pi_{B^{\perp}}$.

Therefore, one defines the oblique projection of a matrix $A$ along the row space of the matrix $B$ onto the row space of the matrix :

$$
A /{ }_{B} C=\left[A / B^{\perp}\right]\left[C / B^{\perp}\right]^{+} \cdot C=A \Pi_{B^{\perp}}+\left(C \Pi_{B^{\perp}}\right)^{+} \cdot C
$$

Then, another one defines the properties of the orthogonal and oblique projections as follow:

$$
\begin{aligned}
& A_{x} / A_{x}^{\perp}=0 . \\
& A_{x} / A_{x} C_{x}=0 .
\end{aligned}
$$

N4sid method 1

The identification algorithm N4SID is determined by four steps which include:

Determine the projection

$$
Z_{i}=Y_{i \mid 2 i-1} /\left(\begin{array}{c}
u_{0 \mid i-1} \\
u_{i \mid 2 i-1} \\
Y_{0 \mid i-1}
\end{array}\right)=(\underbrace{L_{i}^{1} \mid}_{l i \times m i} \underbrace{L_{i}^{2} \mid}_{l i \times m i} \underbrace{L_{i}^{3}}_{l i \times l i})\left(\begin{array}{l}
u_{p} \\
u_{f} \\
Y_{p}
\end{array}\right) .
$$


$Z_{i+1}=Y_{i+1 \mid 2 i-1}\left(\begin{array}{c}u_{p}^{+} \\ u_{f}^{-} \\ Y_{p}^{+}\end{array}\right)$

Computing singular value decomposition (SVD)

Let the matrix $\mathrm{M}$ be the rank deficient space which coincides with that of columns $\Gamma_{i}$. Therefore, we compute the singular value decomposition SVD passing through the following mathematical steps:

$$
\begin{aligned}
& M=\left(\begin{array}{ll}
U_{1} & U_{2}
\end{array}\right)\left(\begin{array}{cc}
\sum_{1} & 0 \\
0 & 0
\end{array}\right) V^{t} . \\
& L_{i}^{1}=\Gamma_{i}\left(\left[A^{i}-Q_{i} \Gamma_{i}\right] S\left(R^{-1}\right)_{1 \mid m i}+\Delta_{i}^{d}-Q_{i} H_{i}^{d}\right) . \\
& L_{i}^{2}=H_{i}^{d}+\Gamma_{i}\left(\left[A^{i}-Q_{i} \Gamma_{i}\right] S\left(R^{-1}\right)_{m i+1 \mid 2 m i}\right) . \\
& L_{i}^{3}=\Gamma_{i} Q_{i} .
\end{aligned}
$$

Where $\left(R^{-1}\right)_{1 \mid m i}$ is the sub-matrix of the column 1 to the column mi.

$\left.(\underbrace{L_{i}^{1}}_{l i \times m i} \mid \underbrace{L_{i}^{3}}_{l i \times l i})\right)\left(\begin{array}{l}u_{0 \mid i-1} \\ Y_{0 \mid i-1}\end{array}\right)=\left(\begin{array}{ll}U_{1} & U_{2}\end{array}\right)\left(\begin{array}{cc}\sum_{1} & 0 \\ 0 & 0\end{array}\right) V^{t}$.

$\Gamma_{i-1}=\underline{U_{1}} \Sigma_{1}^{1 / 2}$ where $\underline{U_{1}}$ denote respectively the last $l_{(i-1)}$ rows of $U_{1}$.

Determine the system matrices

We assume that $\Gamma_{i}, \Gamma_{i-1}$ and $\mathrm{n}$ the order of system are determined.

$$
\left\{\begin{array}{l}
Z_{i}=\Gamma_{i} \hat{X}_{i}+H_{i}^{d} U_{i \mid 2 i-1 .} \\
Z_{i+1}=\Gamma_{i-1} \hat{X}_{i+1}+H_{i-1}^{d} U_{i+1 \mid 2 i-1 .}
\end{array}\right.
$$

We can extract:

$$
\left\{\begin{array}{l}
\hat{X}_{i}=\Gamma_{i}^{+}\left(Z_{i}-H_{i}^{d} U_{i \mid 2 i-1}\right) . \\
\hat{X}_{i+1}=\Gamma_{i-1}^{+}\left(Z_{i+1}-H_{i-1}^{d} U_{i+1 \mid 2 i-1 .}\right.
\end{array}\right.
$$

In our case, we need to know $H_{i}^{d}$ and $H_{i-1}^{d}$ from:

$$
\begin{gathered}
\hat{X}_{i+1}=A \hat{X}_{i}+B U_{i \mid i}+\left(\begin{array}{c}
u_{0 \mid 2 i-1} \\
u_{0 \mid i-1} \\
\hat{X}_{i}
\end{array}\right)^{-} \text {and } \\
Y_{i \mid i}=C \hat{X}_{i}+D U_{i \mid i}+\left(\begin{array}{c}
u_{0 \mid 2 i-1} \\
u_{0 \mid i-1} \\
\hat{X}_{i}
\end{array}\right)^{-}
\end{gathered}
$$


Where $(.)^{-}$indicates a matrix whose row space is perpendicular to the row space of (.). Also, we know that:

$$
\left(\frac{\hat{X}_{i+1}}{Y_{i \mid i}}\right)=\left(\frac{A}{C}\right) \hat{X}_{i}+\left(\frac{B}{D}\right) U_{i \mid i}+\left(\begin{array}{l}
u_{0 \mid 2 i-1} \\
Z_{i} \\
\hat{X}_{i}
\end{array}\right)^{-},
$$

Then, if we subtitle the expression for $\hat{X}_{i}$ and $\hat{X}_{i+1}$, we get:

$$
\left(\frac{\Gamma_{i-1}^{+} Z_{i+1}}{Y_{i \mid i}}\right)=\left(\frac{A}{C}\right) \Gamma_{i}^{+} Z_{i}+\left(\begin{array}{l}
K_{12} \\
K_{22}
\end{array}\right) U_{i \mid 2 i-1}+\left(\begin{array}{l}
U_{0 \mid 2 i-1} \\
Z_{i} \\
\hat{X}_{i}
\end{array}\right)^{-},
$$

where we define:

$$
\left(\frac{K_{12}}{K_{22}}\right)=\left[\frac{B-A \Gamma_{i}^{+}\left(\begin{array}{l}
D \\
\Gamma_{i-1} B
\end{array}\right) \Gamma_{i-1}^{+} H_{i-1}^{d}-A \Gamma_{i}^{+}\left(\begin{array}{l}
0 \\
H_{i}^{d}
\end{array}\right)}{D-C \Gamma_{i}^{+}\left(\begin{array}{l}
D \\
\Gamma_{i-1} B
\end{array}\right)-A \Gamma_{i}^{+}\left(\begin{array}{l}
0 \\
H_{i}^{d}
\end{array}\right)}\right] .
$$

We observe that the matrices $\mathrm{B}$ and $\mathrm{D}$ appear linearly in the matrices $K_{12}, K_{22}$. From the last equation, we obtain:

$$
\left(\frac{\Gamma_{i-1}^{+} Z_{i+1}}{Y_{i \mid i}}\right) / \Pi=\left(\frac{A K_{12}}{C K_{22}}\right)\left(\frac{\Gamma_{i}^{+} Z_{i}}{U_{i \mid 2 i-1}}\right) / \Pi .
$$

The unknown in this set of linear equations are $, C, K_{12}, K_{22}$. The solution is to compute the least mean square:

$$
\begin{aligned}
& \min _{A, C, K_{12}, K_{22}}\left\|\left(\frac{\Gamma_{i-1}^{+} Z_{i+1}}{Y_{i \mid i}}\right)-\left(\frac{A K_{12}}{C K_{22}}\right)\left(\frac{\Gamma_{i}^{+} Z_{i}}{U_{i \mid 2 i-1}}\right)\right\|_{F}{ }^{2} \text { with } \\
& \left(\frac{\Gamma^{+}{ }_{i-1} Z_{i+1}}{Y_{i \mid i}}\right)=\left(\begin{array}{ll}
K_{11} & K_{12} \\
K_{21} & K_{22}
\end{array}\right) \times\left(\frac{\Gamma_{i}^{+} Z_{i}}{U_{i \mid 2 i-1}}\right)+\left(\begin{array}{c}
p_{v} \\
p_{w}
\end{array}\right),
\end{aligned}
$$

where $p_{v}, p_{w}$ are residues.

The system matrix $A$ is equal to $K_{11}$ and $C$ is equal to $K_{21}$. Then we compute the matrices $\mathrm{B}$ and D from $K_{12}, K_{22}, A$ and $C$ by solving a set of linear equations. Finally, we compute the covariance's matrices as follows:

$$
\left(\begin{array}{cc}
Q^{s} & S^{s} \\
\left(S^{s}\right)^{t} & R^{s}
\end{array}\right) \leftarrow \frac{1}{j}\left(\begin{array}{ll}
p_{v} p_{v}^{t} & p_{v} p_{w}^{t} \\
p_{w} p_{v}^{t} & p_{w} p_{w}^{t}
\end{array}\right) .
$$

N4sid method 2 
The N4SID2 identification algorithm could also be determined by the next four steps which are: 1-Determine the projections:

$$
\begin{aligned}
Z_{i+1} & =Y_{i+1 \mid 2 i-1} /\left(\begin{array}{c}
u_{0 \mid i-1} \\
u_{i+1 \mid 2 i-1} \\
Y_{0 \mid i}
\end{array}\right) . \\
Z_{i+1} & =Y_{i+1 \mid 2 i-1}\left(\begin{array}{c}
u_{0 \mid i-1} \\
u_{i+1 \mid 2 i-1} \\
Y_{0 \mid i}
\end{array}\right) . \\
Z_{i+1} & =Y_{i+1 \mid 2 i-1} /\left(\begin{array}{c}
u_{0 \mid i-1} \\
u_{i+1 \mid 2 i-1} \\
Y_{0 \mid i}
\end{array}\right) . \\
& =(\underbrace{L_{i+1}^{1}}_{l(i-1) \times m(i+1)}|\underbrace{L_{i+1}^{2}}_{l(i-1) \times m(i-1)}| \underbrace{L_{i+1}^{3}}_{l(i-1) \times l(i+1)})\left(\begin{array}{c}
u_{p}^{+} \\
u_{f}^{-} \\
Y_{p}^{+}
\end{array}\right) .
\end{aligned}
$$

2-Compute singular value decomposition (SVD)

$$
(\underbrace{L_{i}^{1}}_{l i \times m i} \mid \underbrace{L_{i}^{3}}_{l i \times l i})\left(\begin{array}{l}
u_{0 \mid i-1} \\
Y_{0 \mid i-1}
\end{array}\right)=\left(\begin{array}{ll}
U_{1} & U_{2}
\end{array}\right)\left(\begin{array}{cc}
\Sigma_{1} & 0 \\
0 & 0
\end{array}\right) V^{t} .
$$

The order of the system is equal to the number of non-zero singular values.

$$
\Gamma_{i}=U_{1} \Sigma_{1}^{1 / 2} \text { and } \Gamma_{i-1}=U_{1} \Sigma_{1}^{1 / 2} .
$$

3-Estimate the states $\tilde{X}_{i}$ and $\tilde{X}_{i+1}$

$$
\begin{aligned}
& \tilde{X}_{i}=\Gamma_{i}^{+}(\underbrace{L_{i}^{1}}_{l i \times m i} \mid \underbrace{L_{i}^{3}}_{l i \times l i})\left(\begin{array}{l}
u_{0 \mid i-1} \\
Y_{0 \mid i-1}
\end{array}\right) . \\
& \tilde{X}_{i+1}=\Gamma_{i}^{+}(\underbrace{L_{i+1}^{1}}_{l(i-1) \times m(i+1)} \mid \underbrace{L_{i+1}^{3}}_{i(i-1) \times l(i+1)})\left(\begin{array}{c}
u_{0 \mid i} \\
Y_{0 \mid i}
\end{array}\right) .
\end{aligned}
$$

4-Extract system matrices from least square solution

$$
\left(\frac{\tilde{X}_{i+1}}{Y_{i \mid i}}\right)=\left(\begin{array}{cc}
\tau_{11} & \tau_{12} \\
\tau_{21} & \tau_{22}
\end{array}\right) \times\left(\frac{\tilde{X}_{i}}{U_{i \mid i}}\right)+\left(\begin{array}{c}
p_{v} \\
p_{w}
\end{array}\right) .
$$




$$
\begin{aligned}
& \left(\begin{array}{ll}
A & B \\
C & D
\end{array}\right) \leftarrow\left(\begin{array}{ll}
\overline{\tau_{11}} & \overline{\tau_{12}} \\
\overline{\tau_{21}} & \overline{\tau_{22}}
\end{array}\right) . \\
& \left(\begin{array}{cc}
Q^{s} & S^{s} \\
\left(S^{s}\right)^{t} & R^{s}
\end{array}\right) \leftarrow \frac{1}{j}\left(\begin{array}{ll}
p_{v} p_{v}^{t} & p_{v} p_{w}^{t} \\
p_{w} p_{v}^{t} & p_{w} p_{w}^{t}
\end{array}\right) .
\end{aligned}
$$

\section{References}

BORJAS, S. D. M. \& GARCIA, C. 2011. Subspace identification for industrial processes TEMA Trends in Applied and Computational Mathematics, 12, 183-194.

BOYD, S., EL GHAOUI, L., FERON, E. \& BALAKRISHNAN, V. 1994. Linear matrix inequalities in system and control theory, Siam.

CAO, K., GAO, X. Z., LAM, H.-K., VASILAKOS, A. V. \& PEDRYCZ, W. 2014. A New Relaxed Stability Condition for Takagi-Sugeno Fuzzy Control Systems Using Quadratic Fuzzy Lyapunov Functions and Staircase Membership Functions. International Journal of Fuzzy Systems, 16, 327-337.

CHADLI, M., MAQUIN, D. \& RAGOT, J. 2002. An LMI formulation for output feedback stabilization in multiple model approach. 41st IEEE Conference on Decision and Control. Las Vegas, NV, USA.

CHANG, X.-H. \& YANG, G.-H. 2010. Relaxed stabilization conditions for continuous-time Takagi-Sugeno fuzzy control systems. Elsevier Information Sciences, 180, 3273-3287.

COstA, A. G., RIUL, J. A. \& MONTENEGRO, P. H. M. 2016. Application of the subspace identification method using the N4SID technique for a robotic manipulator. IEEE Latin America Transactions, 14, 1588-1593.

DE SIMONE, M. C. \& GUIDA, D. 2018. Identification and Control of a Unmanned Ground Vehicle By using Arduino. UPB Scientific Bulletin, Series D: Mechanical Engineering, 80, 141-154.

EUNTAI, K. \& HEEJIN, L. 2000. New approaches to relaxed quadratic stability condition of fuzzy control systems. IEEE Transactions on Fuzzy Systems, 8, 523-534.

HACHICHA, S., KHARRAT, M. \& CHAARI, A. 2014. N4SID and MOESP Algorithms to Highlight the Illconditioning into Subspace Identification. International Journal of Automation and Computing, 11, 30 38.

ICHALAL, D., MARX, B., RAGOT, J. \& MAQUIN, D. 2014. Fault detection, isolation and estimation for Takagi-Sugeno nonlinear systems. Elsevier Journal of the Franklin Institute, 351, 3651-3676.

KISHORE, D., SMRUTI RAJAN, S., ANAND KISHORE, K. \& PANDA, R. C. 2018. An improved identification and control of $3 \times 3$ multi-input multi-output system using relay and subspace method. $J$ Indian Chemical Engineer, 11, 1-15.

KROKAVEC, D. \& FILASOVA, A. 2014. LMI based fuzzy observer design for Takagi-Sugeno models containing vestigial nonlinear terms. Archives Of Control Sciences, 24, 39-52.

LARIMORE, W. E. Canonical variate analysis in identification, filtering, and adaptive control. 29th Conference on Decision and Control, 1990 Honolulu, HI, USA, USA. IEEE, 596-604.

LJUNG, L. 1999. System identification : theory for the user, Upper Saddle River, NJ, Prentice Hall; 2 edition.

MA, Y., LIU, J., LIU, H. \& ZHAO, S. 2018. Active-Reactive Additional Damping Control of a Doubly-Fed Induction Generator Based on Active Disturbance Rejection Control. MDPI Energies, 11, 1314.

MUHAMMAD, M., BUYAMIN, S., AHMAD, M. \& NAWAWI, S. 2013a. Takagi-Sugeno fuzzy modeling of a two-wheeled inverted pendulum robot. Journal of Intelligent and Fuzzy Systems: Applications in Engineering and Technology, 25, 535-546.

MUHAMMAD, M., BUYAMIN, S., AHMAD, M., NAWAWI, S. \& AHMAD, A. 2013b. A new approach to stabilization of continuous time TS fuzzy control systems via fuzzy Lyapunov function. IJMME-IJENS International Journal of Mechanical and Mechatronics Engineering, 13.

NGUYEN, A.-T., SENTOUH, C. \& POPIEUL, J.-C. 2017a. Driver-automation cooperative approach for shared steering control under multiple system constraints: Design and experiments. IEEE Transactions on Industrial Electronics, 64, 3819-3830.

NGUYEN, A.-T., SENTOUH, C. \& POPIEUL, J.-C. 2017b. Fuzzy steering control for autonomous vehicles under actuator saturation: Design and experiments. Elsevier Journal of the Franklin Institute, 335, 93749395.

NGUYEN, A.-T., SENTOUH, C. \& POPIEUL, J.-C. 2018. Sensor Reduction for Driver-Automation Shared Steering Control via an Adaptive Authority Allocation Strategy. IEEE/ASME Transactions on Mechatronics, 23, 5-16. 
PRÍVARA, S., CIGLER, J., VÁŇA, Z., FERKL, L. \& ŠEBEK, M. Subspace identification of poorly excited industrial systems. In: IEEE, ed. 49th Conference on Decision and Control (CDC), 2010 Atlanta, GA, USA. IEEE, 4405-4410.

SENTOUH, C., NGUYEN, A.-T., BENLOUCIF, M. A. \& POPIEUL, J.-C. 2018. Driver-automation cooperation oriented approach for shared control of lane keeping assist systems. IEEE Transactions on Control Systems Technology, 1-17.

SHAFIEEZADEH-ABADEH, S. \& KALHOR, A. 2016. Evolving Takagi-Sugeno model based on online Gustafson-Kessel algorithm and kernel recursive least square method. Springer Evolving Systems, 7, 114.

SÖDERSTRÖM, T. \& STOICA, P. 1989. System identification, Prentice-Hall.

SOFIANOS, N. A. \& BOUTALIS, Y. S. 2014. Multiple models fuzzy control: a redistributed fixed models based approach. Taylor and Francis, Intelligent Automation and Soft Computing, 20, 229-243.

TANAKA, K., NISHIMURA, M. \& WANG, H. O. Multi-objective fuzzy control of high rise/high speed elevators using LMIs. American Control Conference, 1998 Philadelphia, PA, USA, USA. IEEE, 34503454.

VAN OVERSCHEE, P. \& DE MOOR, B. 1994. N4SID: Subspace algorithms for the identification of combined deterministic-stochastic systems. Elsevier Automatica, 30, 75-93.

VAN OVERSCHEE, P. \& DE MOOR, B. L. 1996. Subspace Identification for Linear Systems, Theory Implementation - Applications, Kluwer Academic Publishers.

VERHAEGEN, M. 1994. Identification of the deterministic part of MIMO state space models given in innovations form from input-output data. Elsevier Automatica, 30, 61-74.

YU, C., LJUNG, L. \& VERHAEGEN, M. 2018. Identification of structured state-space models. Elsevier Automatica, 90, 54-61.

ZHANG, M., SHI, P., MA, L., CAI, J. \& SU, H. 2018a. Quantized feedback control of fuzzy Markov jump systems. IEEE transactions on cybernetics, 1-10.

ZHANG, Z., SHI, Y., ZHANG, Z. \& YAN, W. 2018b. New Results on Sliding-Mode Control for Takagi-Sugeno Fuzzy Multiagent Systems. IEEE Transactions on Cybernetics, 1-13. 